\title{
Invariant Measures, Matching and the Frequency of 0 for Signed Binary Expansions
}

\author{
by \\ Karma DAJANI and Charlene KALLE
}

\begin{abstract}
We introduce a family of maps $\left\{S_{\eta}\right\}_{\eta \in[1,2]}$ defined on $[-1,1]$ by $S_{\eta}(x)=2 x-d \eta$, where $d \in\{-1,0,1\}$. Each map $S_{\eta}$ generates signed binary expansions, i.e., binary expansions with digits $-1,0$ and 1 . We study the frequency of the digit 0 in typical expansions as a function of the parameter $\eta$. The transformations $S_{\eta}$ have an ergodic invariant measure $\mu_{\eta}$ that is absolutely continuous with respect to Lebesgue measure. The frequency of the digit 0 is related to the measure $\mu_{\eta}\left(\left[-\frac{1}{2}, \frac{1}{2}\right]\right)$ by the ergodic theorem. We show that the density of $\mu_{\eta}$ is a step function except for a set of parameters of zero Lebesgue measure and full Hausdorff dimension and we give a full description of the maximal parameter intervals on which the density has the same number of steps. We give an explicit formula for the frequency of the digit 0 in typical signed binary expansions on each of these parameter intervals and show that this frequency depends continuously on the parameter $\eta$. Moreover, it takes the value $\frac{2}{3}$ only on the interval $\left[\frac{6}{5}, \frac{3}{2}\right]$ and it is strictly less than $\frac{2}{3}$ on the remainder of the parameter space.
\end{abstract}

2010 Mathematics Subject Classification: 37E05, 28D05, 37E15, 37A45, 37A05.

Keywords: Symmetric doubling maps, binary expansions, Nakada's $\alpha$-continued fractions, matching, interval maps, invariant measures, digit frequency.

\section{§1. Introduction}

Binary expansions are used in a variety of applications. The amount of different representations that numbers have in base 2 depends on the choice of digit set. For example, every integer $n \geq 1$ has a unique expansion of the form

$$
n=\sum_{k=0}^{m} b_{k} 2^{k}, \quad b_{k} \in\{0,1\} \text { and } b_{m} \neq 0,
$$

Communicated by T. Kumagai. Received March 11, 2019. Revised July 26, 2019.

K. Dajani: Department of Mathematics, Utrecht University, P.O. Box 80010, 3508TA Utrecht, The Netherlands;

e-mail: k.dajani1@uu.nl

Mathematisch Instituut, Leiden University, Niels Bohrweg 1, 2333CA Leiden, The Netherlands; e-mail: kallecccj@math.leidenuniv.nl 
but there are infinitely many ways to write $n$ as a signed binary expansion of the form

$$
n=\sum_{k=0}^{m} b_{k} 2^{k}, \quad b_{k} \in\{-1,0,1\} \text { and } b_{m} \neq 0 .
$$

This last property can be useful for technological applications, since it provides the freedom to choose an expansion from a collection of different representations of the same number. For example, from the perspective of public key cryptography using elliptic curves there is an interest in expansions of integers having the lowest number of non-zero digits (see for example [MO90, KT93, CMO98]). For digit set $\{-1,0,1\}$ this is equivalent to having the lowest possible sum $\sum_{k=0}^{m}\left|b_{k}\right|$ or lowest Hamming weight. Out of all possible expansions of the form (1) the minimal weight or separated sign binary expansion is the unique expansion satisfying $b_{k} b_{k+1}=0$ for all $0 \leq k \leq m-1$.

In [DKL06] a dynamical viewpoint to study minimal weight binary expansions was proposed. Instead of considering the finite strings of digits from $\{-1,0,1\}$ appearing in (1), the authors look at the subset $\mathcal{K}$ of $\{-1,0,1\}^{\mathbb{N}}$ of which the language is given by the minimal weight expansions of integers under the dynamics of the shift map $\sigma$. They prove that the frequency of the digit 0 in a typical sequence from $\mathcal{K}$ is $\frac{2}{3}$.

The dynamical system $(\mathcal{K}, \sigma)$ is shown in [DKL06] to be conjugated to the action on the interval $\left[-\frac{2}{3}, \frac{2}{3}\right]$ of the map $T$ given by $T(x)=2 x-b(x)$, where $b(x)=-1$ if $x \in\left[-\frac{2}{3},-\frac{1}{3}\right), b(x)=0$ if $x \in\left[-\frac{1}{3}, \frac{1}{3}\right)$ and $b(x)=1$ if $x \in\left[\frac{1}{3}, \frac{2}{3}\right]$. In this paper we extend the viewpoint from [DKL06] by introducing a one-parameter family of maps $\left\{S_{\eta}:[-1,1] \rightarrow[-1,1]\right\}_{\eta \in[1,2]}$ defined as follows. For each $x \in[-1,1]$ set the digit

$$
d_{1}(x)= \begin{cases}-1 & \text { if } x<-\frac{1}{2} \\ 0 & \text { if } x \in\left[-\frac{1}{2}, \frac{1}{2}\right], \\ 1 & \text { if } x>\frac{1}{2}\end{cases}
$$

and define for each $\eta \in[1,2]$ the map

$$
S_{\eta}(x)=2 x-d_{1}(x) \eta .
$$

We call the maps $S_{\eta}$ symmetric doubling maps. Figure 1 shows the graph of $S_{\eta}$ for various $\eta$. The map $T$ from [DKL06] is isomorphic to the map $S_{\frac{3}{2}}$ after rescaling of the digits. Since we deal with a family of transformations instead of a single map we choose to rescale the digits rather than the domain of the maps, so that all transformations $S_{\eta}$ are defined on the same interval. 


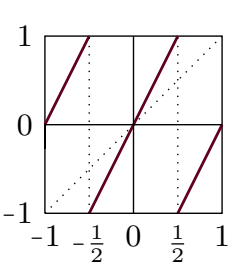

(a) $\eta=2$

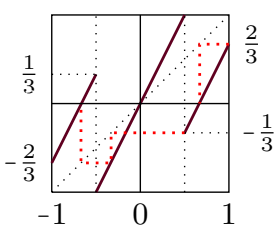

(d) $\eta=\frac{4}{3}$

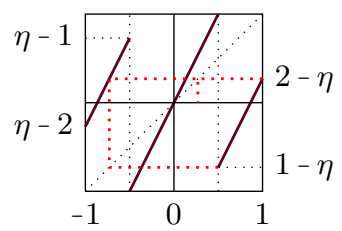

(b) $\eta=\sqrt{3}$

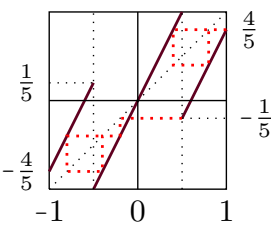

(e) $\eta=\frac{6}{5}$

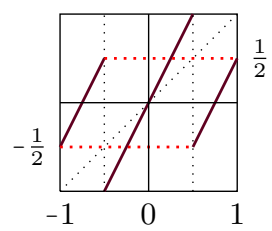

(c) $\eta=\frac{3}{2}$

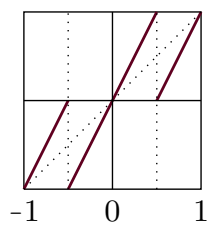

(f) $\eta=1$

Figure 1. The symmetric doubling map for various values of $\eta$. The red dotted lines indicate the orbits of 1 and $1-\eta$.

If we now set for each $n \geq 1$ and $x \in[-1,1]$ the digit $d_{\eta, n}(x)=d_{1}\left(S_{\eta}^{n-1}(x)\right)$, then we can write

$$
S_{\eta}^{n}(x)=2 S_{\eta}^{n-1}(x)-d_{\eta, n}(x) \eta
$$

and obtain a signed binary expansion of $x$ by

$$
x=\sum_{n \geq 1} \frac{d_{\eta, n}(x) \eta}{2^{n}}=\eta \sum_{n \geq 1} \frac{d_{\eta, n}(x)}{2^{n}},
$$

or equivalently by the sequence $\left(d_{\eta, n}(x)\right)_{n \geq 1}$. The digit 0 occurs in position $n$ precisely when $S_{\eta}^{n-1}(x) \in\left[-\frac{1}{2}, \frac{1}{2}\right]$. It follows easily from the literature (see for example [Kop90]) that each map $S_{\eta}$ with $\eta \neq 1$ has a unique ergodic invariant measure $\mu_{\eta}$ absolutely continuous with respect to Lebesgue measure. Then by Birkhoff's ergodic theorem the frequency of the digit 0 in almost all of the corresponding signed binary expansions equals the $\mu_{\eta}$-measure of the interval $\left[-\frac{1}{2}, \frac{1}{2}\right]$. The goal of this article is to find a good expression for the invariant probability density $f_{\eta}$ of $\mu_{\eta}$, so that we can calculate $\mu_{\eta}\left(\left[-\frac{1}{2}, \frac{1}{2}\right]\right)$ and study its dependence on $\eta$.

Results from [Kop90] imply that in general $f_{\eta}$ is an infinite sum of indicator functions, but that $f_{\eta}$ becomes a step function if the map $S_{\eta}$ exhibits the dynamical phenomenon of matching. Matching for interval maps has received quite a lot of 
attention recently, especially in the case of continued fraction transformations; see [NN08, DKS09, CMPT10, CT12, KSS12, BCIT13, BSORG13, CT13, CM18, BCK17, BCMP19] for example. It is the property that for each critical point the orbits of the left and right limits meet after a finite number of iterations and that the derivatives of both orbits are also equal at that time. Due to symmetry and the constant slope, for our family of maps $\left\{S_{\eta}:[-1,1] \rightarrow[-1,1]\right\}_{\eta \in[1,2]}$ we say that $S_{\eta}$ has matching at time $m$ if $m \geq 1$ is the minimal number of iterations, such that

$$
S_{\eta}^{m}(1)=S_{\eta}^{m+1}\left(\frac{1}{2}^{-}\right)=S_{\eta}^{m+1}\left(\frac{1}{2}^{+}\right)=S_{\eta}^{m}(1-\eta) .
$$

The exponent $m$ is called the matching index of $S_{\eta}$. In this article we give a complete description of the matching behaviour of the family $\left\{S_{\eta}:[-1,1] \rightarrow\right.$ $[-1,1]\}_{\eta \in[1,2]}$, i.e., we prove the following.

Theorem A. Up to a set of zero Lebesgue measure and full Hausdorff dimension, the parameter space $[1,2]$ is divided into intervals of parameters $\eta$ on which the density of the absolutely continuous invariant measure $\mu_{\eta}$ for $S_{\eta}$ is a step function with the same number of jumps. Maximal intervals with this property are uniquely determined by the initial parts of the sequences $\left(d_{\eta, n}(1)\right)_{n \geq 1}$ for $\eta$ in the interval.

The maximal intervals from Theorem A are called matching intervals. If the matching index on a matching interval is $m$, then for any $\eta, \eta^{\prime}$ in it we have $d_{\eta, n}(1)=d_{\eta^{\prime}, n}(1)$ for all $1 \leq n \leq m$. By close investigation of the density function of the measure $\mu_{\eta}$, we get the following result.

Theorem B. The map $\eta \mapsto \mu_{\eta}\left(\left[-\frac{1}{2}, \frac{1}{2}\right]\right)$ is continuous on $[1,2]$ and monotone on each matching interval. More precisely, if $J \subseteq[1,2]$ is a matching interval on which the matching index is $m$, then for all $\eta \in J$,

$$
\mu_{\eta}\left(\left[-\frac{1}{2}, \frac{1}{2}\right]\right)=\frac{2^{m-1}}{2^{m}-1}\left(\frac{c}{\eta}+K\right),
$$

where $c$ and $K$ are explicitly given constants depending only on $d_{\eta, n}(1), 1 \leq n \leq$ $m-1$.

On the maximal value of $\mu_{\eta}\left(\left[-\frac{1}{2}, \frac{1}{2}\right]\right)$ and the frequency of the digit 0 in the signed binary expansions from (4) we have the following result.

Theorem C. It holds that $\mu_{\eta}\left(\left[-\frac{1}{2}, \frac{1}{2}\right]\right) \leq \frac{2}{3}$ for any $\eta \in[1,2]$ and $\mu_{\eta}\left(\left[-\frac{1}{2}, \frac{1}{2}\right]\right)=$ $\frac{2}{3}$ if and only if $\eta \in\left[\frac{6}{5}, \frac{3}{2}\right]$. Equivalently, for any $\eta \in[1,2]$ the frequency of the digit 0 in a typical signed binary expansion obtained from the map $S_{\eta}$ is at most $\frac{2}{3}$ and this maximum is obtained for typical expansions if and only if $\eta \in\left[\frac{6}{5}, \frac{3}{2}\right]$. 
This theorem extends the results from [DKL06], where the authors obtained that $\mu_{\frac{3}{2}}\left(\left[-\frac{1}{2}, \frac{1}{2}\right]\right)=\frac{2}{3}$. It is also the analogue in the context of symmetric doubling maps of the first question mentioned in [KSS12] in relation to the maximality of the entropy of Nakada's $\alpha$-continued fraction maps. Theorem $\mathrm{C}$ gives a positive answer for the piecewise linear analogue.

An ingredient in the proof of these results is a correspondence between the maps $S_{\eta}$ and Nakada's $\alpha$-continued fraction maps. As an immediate consequence of this correspondence we obtain a lot of information on the subset $\mathcal{N} \subseteq[1,2]$ of those parameters $\eta$ for which $S_{\eta}$ does not have matching. In particular we will see that, besides being a Lebesgue null set of full Hausdorff dimension, $\mathcal{N}$ is also totally disconnected and closed and that to each matching interval for the family $\left\{S_{\eta}\right\}_{\eta \in[1,2]}$ there corresponds an element in the set $\mathcal{N}$ that is transcendental. Moreover, the set $\mathcal{N}$ is related to interesting subsets of the parameter space of several other one-parameter families of dynamical systems as explained in Remark 3.2.

The article is organised as follows. In the second section we prove that $S_{\eta}$ has matching for Lebesgue almost all $\eta \in[1,2]$. In the third section we describe the connection between the symmetric doubling maps and the $\alpha$-continued fraction maps and prove Theorem A. In the fourth section we closely examine the density function $f_{\eta}$ on the matching intervals and prove Theorem B. In the last section we prove Theorem C.

\section{§2. First results on matching and the frequency of 0}

\section{$\S 2.1$. The invariant density}

The frequency of the digit 0 in the signed binary expansions produced by the symmetric doubling map $S_{\eta}, \eta \in[1,2]$, from (2) can be obtained from Birkhoff's ergodic theorem with an explicit expression for the density function of an absolutely continuous invariant measure. For $\eta=1$ (see Figure $1(\mathrm{f})$ ), the map $S_{\eta}$ has two ergodic components given by Lebesgue measure concentrated on the left and right halves of the interval. Under any convex combination of these ergodic components the frequency of the digit 0 in typical signed binary expansions equals $\frac{1}{2}$. Since all is known in this case, we will not consider $\eta=1$ further.

To obtain absolutely continuous invariant measures of the maps $S_{\eta}$ we invoke the results from [Kop90]. These results apply to a class of piecewise linear maps defined on the interval $[0,1]$ and with the property that the end points 0 and 1 are mapped into the set $\{0,1\}$. The article [Kop90] is completely devoted to giving a formula for the density functions of absolutely continuous invariant measures of such maps by verifying that these densities are fixed points of the Perron-Frobenius 
operator. To comply with these conditions we follow the procedure from [Kop90] to a scaled, shifted and extended version of $S_{\eta}$, namely to the map $F:[0,1] \rightarrow[0,1]$ (see Figure 2) given by

$$
F(x)= \begin{cases}2 x & \text { if } x \in\left[0, \frac{1}{2}-\frac{1}{4 \eta}\right) \\ 2 x-\frac{1}{2} & \text { if } x \in\left[\frac{1}{2}-\frac{1}{4 \eta}, \frac{1}{2}+\frac{1}{4 \eta}\right] \\ 2 x-1 & \text { if } x \in\left(\frac{1}{2}+\frac{1}{4 \eta}, 1\right]\end{cases}
$$

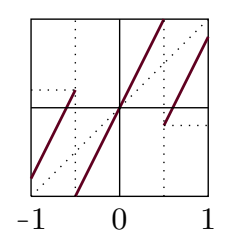

(a) $S_{\eta}$

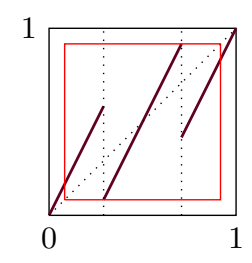

(b) $F$

Figure 2. The extended and shifted version $F$ of a map $S_{\eta}$. The restriction of $F$ to the red box is essentially the map $S_{\eta}$ on the left and any absolutely continuous invariant measure of $F$ is supported inside this box.

For $F$ it holds that $F(1-x)=1-F(x)$. Using the notation from [Kop90], let the points $a_{1}, a_{2}, b_{1}$ and $b_{2}$ be the images of the critical points of $F$, i.e.,

$$
\begin{array}{ll}
a_{1}=2\left(\frac{1}{2}-\frac{1}{4 \eta}\right)=1-\frac{1}{2 \eta}, & b_{1}=2\left(\frac{1}{2}-\frac{1}{4 \eta}\right)-\frac{1}{2}=\frac{1}{2}-\frac{1}{2 \eta}, \\
a_{2}=2\left(\frac{1}{2}+\frac{1}{4 \eta}\right)-\frac{1}{2}=\frac{1}{2}+\frac{1}{2 \eta}=1-b_{1}, & b_{2}=2\left(\frac{1}{2}+\frac{1}{4 \eta}\right)-1=\frac{1}{2 \eta}=1-a_{1} .
\end{array}
$$

The critical points divide the unit interval into three pieces, $I_{1}=\left[0, \frac{1}{2}-\frac{1}{4 \eta}\right)$, $I_{2}=\left[\frac{1}{2}-\frac{1}{4 \eta}, \frac{1}{2}+\frac{1}{4 \eta}\right]=1-I_{2}$ and $I_{3}=\left(\frac{1}{2}+\frac{1}{4 \eta}, 1\right]=1-I_{1}$. As in [Kop90] define

$$
\mathrm{KI}_{n}(y)=\sum_{t \geq 0} \frac{1}{2^{t+1}} 1_{I_{n}}\left(F^{t}(y)\right) \text {. }
$$

Then due to symmetry,

$$
\begin{array}{lll}
\mathrm{KI}_{1}\left(a_{1}\right)=\mathrm{KI}_{3}\left(b_{2}\right), & \mathrm{KI}_{2}\left(a_{1}\right)=\mathrm{KI}_{2}\left(b_{2}\right), & \mathrm{KI}_{3}\left(a_{1}\right)=\mathrm{KI}_{1}\left(b_{2}\right), \\
\mathrm{KI}_{1}\left(a_{2}\right)=\mathrm{KI}_{3}\left(b_{1}\right), & \mathrm{KI}_{2}\left(a_{2}\right)=\mathrm{KI}_{2}\left(b_{1}\right), & \mathrm{KI}_{3}\left(a_{2}\right)=\mathrm{KI}_{1}\left(b_{1}\right)
\end{array}
$$


Let $M=\left(\mu_{i, j}\right)$ be the $3 \times 2$ matrix with entries

$$
\begin{aligned}
& \mu_{1,1}=\frac{1}{2}+\frac{1}{2} \mathrm{KI}_{1}\left(a_{1}\right)-\frac{1}{2} \mathrm{KI}_{1}\left(b_{1}\right)=\frac{1}{2}+\frac{1}{2} \mathrm{KI}_{3}\left(a_{2}\right)-\frac{1}{2} \mathrm{KI}_{3}\left(b_{2}\right)=-\mu_{3,2}, \\
& \mu_{2,1}=-\frac{1}{2}+\frac{1}{2} \mathrm{KI}_{2}\left(a_{1}\right)-\frac{1}{2} \mathrm{KI}_{2}\left(b_{1}\right)=-\frac{1}{2}+\frac{1}{2} \mathrm{KI}_{2}\left(b_{2}\right)-\frac{1}{2} \mathrm{KI}_{2}\left(a_{2}\right)=-\mu_{2,2}, \\
& \mu_{3,1}=\frac{1}{2} \mathrm{KI}_{3}\left(a_{1}\right)-\frac{1}{2} \mathrm{KI}_{3}\left(b_{1}\right)=\frac{1}{2} \mathrm{KI}_{1}\left(b_{2}\right)-\frac{1}{2} \mathrm{KI}_{1}\left(a_{2}\right)=-\mu_{1,2},
\end{aligned}
$$

so $M$ has the form

$$
M=\left(\begin{array}{l}
a-c \\
b-b \\
c-a
\end{array}\right) .
$$

From [Kop90, Lem. 1] we obtain that, for each $y \in[0,1]$,

$$
\frac{1}{2} \mathrm{KI}_{2}(y)+\mathrm{KI}_{3}(y)=y \quad \text { and } \quad \mathrm{KI}_{1}(y)+\mathrm{KI}_{2}(y)+\mathrm{KI}_{3}(y)=1 .
$$

Then (5) gives for $j=1,2$,

$$
\begin{aligned}
\frac{1}{2} \mu_{2, j}+\mu_{3, j} & =-\frac{1}{4}+\frac{1}{4} \mathrm{KI}_{2}\left(a_{j}\right)-\frac{1}{4} \mathrm{KI}_{2}\left(b_{j}\right)+\frac{1}{2} \mathrm{KI}_{3}\left(a_{j}\right)-\frac{1}{2} \mathrm{KI}_{3}\left(b_{j}\right) \\
& =-\frac{1}{4}+\frac{1}{2} a_{j}-\frac{1}{2} b_{j}=0 .
\end{aligned}
$$

From (6) we get $a=c=-\frac{b}{2}$, so that

$$
M=\left(\begin{array}{cc}
-\frac{b}{2} & \frac{b}{2} \\
b & -b \\
-\frac{b}{2} & \frac{b}{2}
\end{array}\right) .
$$

According to [Kop90, Thm. 1] an invariant density for $F$ is given by

$$
h(x)=\sum_{n \geq 0} \frac{1}{2^{n+1}}\left(1_{\left[0, F^{n} a_{1}\right)}(x)-1_{\left[0, F^{n} b_{1}\right)}(x)+1_{\left[0, F^{n} a_{2}\right)}(x)-1_{\left[0, F^{n} b_{2}\right)}(x)\right) .
$$

When translated back to the map $S_{\eta}$, this gives the probability density

$$
\begin{aligned}
f_{\eta}(x)=\frac{1}{C} \sum_{n \geq 0} \frac{1}{2^{n+1}}( & 1_{\left[-1, S_{\eta}^{n}(\eta-1)\right)}(x)-1_{\left[-1, S_{\eta}^{n}(-1)\right)}(x) \\
& \left.+1_{\left[-1, S_{\eta}^{n}(1)\right)}(x)-1_{\left[-1, S_{\eta}^{n}(1-\eta)\right)}(x)\right),
\end{aligned}
$$

where $C$ is a normalising constant. Moreover, [Kop90, Thm. 2] states that there is a one-to-one correspondence between the solutions $\gamma$ of the equation $M \cdot \gamma=0$ and the space of invariant measures of $F$ (and thus of $S_{\eta}$ ). From (7) it follows that the solution space of $M \cdot \gamma=0$ is one-dimensional, so that each $S_{\eta}$ has a unique, hence ergodic, absolutely continuous invariant probability measure. 


\section{§2.2. Matching almost everywhere}

Note that the density function $f_{\eta}$ is an infinite sum of indicator functions over intervals with end points in the set

$$
\left\{S_{\eta}^{n}(1-\eta), S_{\eta}^{n}(1), S_{\eta}^{n}(\eta-1), S_{\eta}^{n}(-1): n \geq 0\right\} .
$$

There are two situations in which the infinite sum becomes a finite sum and the density becomes a step function. Firstly, this happens when the orbits of 1 and $1-\eta$ under $S_{\eta}$ are finite (and thus by symmetry also the orbits of -1 and $\eta-1$ are finite). In that case the set from (9) becomes finite and $S_{\eta}$ has a Markov partition. For a concrete example, consider $\eta=\frac{3}{2}$ from Figure 1(c). A Markov partition is given here by

$$
\left\{\left(-1,-\frac{1}{2}\right),\left(-\frac{1}{2}, \frac{1}{2}\right),\left(\frac{1}{2}, 1\right)\right\} \text {. }
$$

From (3) it follows that the orbits of $S_{\eta}$ are determined as follows:

$$
S_{\eta}^{n}(x)=2^{n} x-d_{\eta, 1}(x) 2^{n-1} \eta-\cdots-d_{\eta, n-1}(x) 2 \eta-d_{\eta, n}(x) \eta .
$$

This description makes it clear that if the orbit of 1 is finite, i.e., if there are $k \neq n$ such that $S_{\eta}^{n}(1)=S_{\eta}^{k}(1)$, then $\eta \in \mathbb{Q}$. Hence, for most $\eta$ a Markov partition does not exist. From (8) we see that $f_{\eta}$ is also a step function if there is an $m \geq 1$, such that $S_{\eta}^{m}(1)=S_{\eta}^{m}(1-\eta)$, i.e., if $S_{\eta}$ has matching. Recall the definition of matching from the introduction:

Definition 2.1. The map $S_{\eta}$ has matching if there is an $m \geq 1$, such that $S_{\eta}^{m}(1)=$ $S_{\eta}^{m}(1-\eta)$. For $S_{\eta}$ we define the matching index $m(\eta)$ by

$$
m(\eta):=\inf \left\{m \geq 1: S_{\eta}^{m}(1)=S_{\eta}^{m}(1-\eta)\right\} .
$$

If $S_{\eta}$ does not have matching, then $m(\eta)=\infty$.

Remark 2.1. (i) As mentioned in the introduction, the usual definition of matching for an interval map $T_{\xi}$ in a one-parameter family $\left\{T_{\xi}\right\}$ is that for each critical point $c$ there are exponents $N, M \geq 1$, such that $T_{\xi}^{N}\left(c^{-}\right)=T_{\xi}^{M}\left(c^{+}\right)$, and also $\left(T_{\xi}^{N}\right)^{\prime}\left(c^{-}\right)=\left(T_{\xi}^{M}\right)^{\prime}\left(c^{+}\right)$where $c^{-}$and $c^{+}$denote the left and right limits to $c$ respectively. The additional condition on the derivative guarantees that around the parameter $\xi$ there is a whole interval of parameters with the same matching exponents $N$ and $M$. Moreover, if the maps $T_{\xi}$ are piecewise smooth, this condition guarantees that the invariant densities of the maps are piecewise smooth (see [BCMP19, Rem. 1]). The derivative of $S_{\eta}^{N}$ equals $2^{N}$. Hence, the map $S_{\eta}$ can have matching according to this definition only if $N=M$. So, our definition of matching from Definition 2.1 for the maps $S_{\eta}$ is consistent with the usual one. 
(ii) Matching does not exclude a Markov partition and vice versa. For $\eta=\frac{6}{5}$ for example (see Figure 1(e)), we have a Markov partition, but we do not have matching. The orbits of 1 and $1-\eta$ are given by

$$
\begin{array}{lll}
S_{\eta}(1)=2-\eta=\frac{4}{5}, & S_{\eta}^{2}(1)=\frac{2}{5}, & S_{\eta}^{3}(1)=S_{\eta}(1), \\
S_{\eta}(1-\eta)=S_{\eta}\left(-\frac{1}{5}\right)=-\frac{2}{5}, & S_{\eta}^{2}(1-\eta)=-\frac{4}{5}, & S_{\eta}^{3}(1-\eta)=S_{\eta}(1-\eta) .
\end{array}
$$

For $\eta=\frac{4}{3}$ (see Figure $1(\mathrm{~d})$ ) there is matching and a Markov partition since $S_{\eta}^{2}(1)=$ $0=S_{\eta}^{2}(1-\eta)$. For $\eta=\sqrt{3}$ (see Figure $1(\mathrm{~b})$ ) we have matching, but no Markov partition. The orbits of 1 and $1-\eta$ are given by

$$
S_{\eta}(1)=2-\sqrt{3} \text { and } S_{\eta}(1-\sqrt{3})=2-2 \sqrt{3}+\sqrt{3}=S_{\eta}(1) .
$$

There is one interval, namely $\left[\frac{3}{2}, 2\right]$, for which we can immediately determine whether matching occurs and compute the frequency of 0 in the sequence $\left(d_{\eta, n}(x)\right)_{n \geq 1}$ for typical $x$. For $\eta=\frac{3}{2}$ we know from Figure 1 (c) that $S_{\eta}$ has a Markov partition. If $\eta \in\left(\frac{3}{2}, 2\right]$, then $1-\eta<-\frac{1}{2}$ and $\eta-1>2-\eta$. This gives that $S_{\eta}(1-\eta)=2(1-\eta)+\eta=2-\eta=S_{\eta}(1)$. Hence, we have matching after one step and we have identified our first matching interval. By (8) we get that for $\eta \in\left[\frac{3}{2}, 2\right]$ the invariant probability density $f_{\eta}$ is given by

$$
f_{\eta}:[-1,1] \rightarrow[-1,1], \quad x \mapsto \frac{1}{2 \eta}\left(1+1_{(1-\eta, \eta-1)}(x)\right) .
$$

So,

$$
\mu_{\eta}\left(\left[-\frac{1}{2}, \frac{1}{2}\right]\right)=\frac{1}{2 \eta} \int_{-\frac{1}{2}}^{\frac{1}{2}} 1+1_{(1-\eta, \eta-1)}(x) d x=\frac{1}{\eta}
$$

which on the interval $\left[\frac{3}{2}, 2\right]$ is maximal for $\eta=\frac{3}{2}$, giving $\mu_{\frac{3}{2}}\left(\left[-\frac{1}{2}, \frac{1}{2}\right]\right)=\frac{2}{3}$. Hence, the maximal frequency of the digit 0 in the sequences $\left(d_{\eta, n}(x)\right)_{n \geq 1}$ for typical $x$ and $\eta \in\left[\frac{3}{2}, 2\right]$ is obtained for $\eta=\frac{3}{2}$ and equals $\frac{2}{3}$.

From now on we let $\eta \in\left(1, \frac{3}{2}\right)$. By Definition 2.1 we have matching at time $m$ if $S_{\eta}^{m}(1)=S_{\eta}^{m}(1-\eta)$ and $S_{\eta}^{n}(1) \neq S_{\eta}^{n}(1-\eta)$ for all $1 \leq n \leq m-1$. The next result says that under iterations of $S_{\eta}$ the points 1 and $1-\eta$ are either a distance $\eta$ apart or mapped to the same point.

Proposition 2.1. For any $n \geq 0, S_{\eta}^{n}(1)-S_{\eta}^{n}(1-\eta) \in\{0, \eta\}$. Moreover, $m(\eta)=m$ if and only if $S_{\eta}^{m-1}(1)>\frac{1}{2}$ and $S_{\eta}^{m-1}(1-\eta)<-\frac{1}{2}$.

Proof. We prove the first statement by induction.

- For $n=0$ it is true, since $1-(1-\eta)=\eta$. 
- Assume now that for some $n \geq 0, S_{\eta}^{n}(1)-S_{\eta}^{n}(1-\eta) \in\{0, \eta\}$. If $S_{\eta}^{n}(1)-$ $S_{\eta}^{n}(1-\eta)=0$, then $S_{\eta}^{k}(1)-S_{\eta}^{k}(1-\eta)=0$ for all $k \geq n$, so assume that $S_{\eta}^{n}(1)-S_{\eta}^{n}(1-\eta)=\eta$. From (10) it follows that there are $b, c \in \mathbb{Z}$ such that

$$
S_{\eta}^{n}(1)=2^{n}-b \eta \quad \text { and } \quad S_{\eta}^{n}(1-\eta)=2^{n}-c \eta .
$$

This implies that $-b \eta+c \eta=\eta$, so $c=1+b$. Since $\eta>1, S_{\eta}^{n}(1)>0$ and $S_{\eta}^{n}(1-\eta)<0$. Moreover, $S_{\eta}^{n}(1)$ and $S_{\eta}^{n}(1-\eta)$ cannot both lie in the interval $\left[-\frac{1}{2}, \frac{1}{2}\right]$. We distinguish three cases.

Case 1: Assume $0<S_{\eta}^{n}(1)<\frac{1}{2}$, so $S_{\eta}^{n}(1-\eta)<-\frac{1}{2}$. Then $S_{\eta}^{n+1}(1)=2^{n+1}-2 b \eta$ and $S_{\eta}^{n+1}(1-\eta)=2^{n+1}-2 c \eta+\eta=2^{n+1}-2(b+1) \eta+\eta=2^{n+1}-2 b \eta-\eta$. Hence, $S_{\eta}^{n+1}(1)-S_{\eta}^{n+1}(1-\eta)=\eta$.

Case 2: Assume $S_{\eta}^{n}(1)>\frac{1}{2}$ and $-\frac{1}{2}<S_{\eta}^{n}(1-\eta)<0$. Then $S_{\eta}^{n+1}(1)=2^{n+1}-$ $2 b \eta-\eta$ and $S_{\eta}^{n+1}(1-\eta)=2^{n+1}-2 c \eta=2^{n+1}-2(b+1) \eta=2^{n+1}-2 b \eta-2 \eta$. So, $S_{\eta}^{n+1}(1)-S_{\eta}^{n+1}(1-\eta)=\eta$.

Case 3: Assume $S_{\eta}^{n}(1)>\frac{1}{2}$ and $S_{\eta}^{n}(1-\eta)<-\frac{1}{2}$. Then $S_{\eta}^{n+1}(1)=2^{n+1}-2 b \eta-\eta$ and $S_{\eta}^{n+1}(1-\eta)=2^{n+1}-2 c \eta+\eta=2^{n+1}-2(b+1) \eta+\eta=2^{n+1}-2 b \eta-\eta$. So, $S_{\eta}^{n+1}(1)-S_{\eta}^{n+1}(1-\eta)=0$ and matching occurs at step $n+1$.

In the case $S_{\eta}^{n}(1)=\frac{1}{2}$, the map $S_{\eta}$ has a Markov partition, since $S_{\eta}^{n}(1-\eta)=\frac{1}{2}-\eta$ and $\eta>1$ imply that $S_{\eta}^{n+1}(1-\eta)=1-2 \eta+\eta=1-\eta$. A similar situation occurs when $S_{\eta}^{n}(1-\eta)=-\frac{1}{2}$ and $S_{\eta}^{n}(1)=\eta-\frac{1}{2}>\frac{1}{2}$. Then $S_{\eta}^{n+1}(1)=2 \eta-1-\eta=\eta-1$. By (2) $S_{\eta}$ does not have matching in these cases and $S_{\eta}^{n}(1)-S_{\eta}^{n}(1-\eta)=\eta$ for all $n \geq 0$. Hence we see that the first statement holds for all $n \in \mathbb{N}$. For the second statement note that the only way in which the difference between $S_{\eta}^{n}(1)$ and $S_{\eta}^{n}(1-\eta)$ becomes 0 is precisely in case 3 from the induction proof.

Remark 2.2. Consider again the situation that $S_{\eta}^{n}(1)=\frac{1}{2}$ or $S_{\eta}^{n}(1-\eta)=-\frac{1}{2}$ for some $n$. Whether or not matching occurs depends on the choice made for the action of $S_{\eta}$ on the critical points. Our choice for the definition from (2) implies that $S_{\eta}$ does not have matching in these cases.

From this proposition we obtain an alternative characterisation of the matching index $m(\eta)$ from Definition 2.1:

$$
m(\eta)=\inf \left\{n \geq 1: \frac{1}{2}<S_{\eta}^{n}(1)<\eta-\frac{1}{2}\right\}+1 .
$$

We now establish a relation between the maps $S_{\eta}$ and the doubling map

$$
D:[0,1] \rightarrow[0,1], \quad x \mapsto 2 x \quad(\bmod 1)
$$


that will be of help later: $D$ has Lebesgue measure as an ergodic invariant measure and it can be used to generate binary expansions of numbers in $[0,1]$ by setting for each $n \geq 1$,

$$
b_{n}(x)= \begin{cases}0 & \text { if } D^{n-1}(x)<\frac{1}{2} \\ 1 & \text { if } D^{n-1}(x) \geq \frac{1}{2}\end{cases}
$$

Then one can write $D^{n}(x)=2 D^{n-1}(x)-b_{n}(x)$ and so $x=\sum_{n \geq 1} \frac{b_{n}(x)}{2^{n}}$. Let $d_{\eta}=\left(d_{\eta, n}\right)_{n \geq 1}=\left(d_{\eta, n}(1)\right)_{n \geq 1}$ denote the digit sequence obtained from $S_{\eta}$ for the point 1 .

Proposition 2.2. Let $1<\eta<\frac{3}{2}$ and let $m$ be the first index such that either $S_{\eta}^{m}(1) \in\left(\frac{1}{2}, \eta-\frac{1}{2}\right)$ or $D^{m}\left(\frac{1}{\eta}\right) \in\left(\frac{1}{2 \eta}, 1-\frac{1}{2 \eta}\right)$. Then $\frac{1}{\eta} S_{\eta}^{n}(1)=D^{n}\left(\frac{1}{\eta}\right)$ and thus $d_{\eta, n}=b_{n}\left(\frac{1}{\eta}\right)$ for all $0 \leq n \leq m$. Moreover, both $S_{\eta}^{m}(1) \in\left(\frac{1}{2}, \eta-\frac{1}{2}\right)$ and $D^{m}\left(\frac{1}{\eta}\right) \in$ $\left(\frac{1}{2 \eta}, 1-\frac{1}{2 \eta}\right)$.

Proof. First note that from the proof of the previous proposition it follows that $S_{\eta}^{n}(1)>0$ for all $0 \leq n<m$. The last statement of the proposition immediately follows from the fact that $\frac{1}{\eta} S_{\eta}^{m}(1)=D^{m}\left(\frac{1}{\eta}\right)$. We prove the first statement by induction.

- For $n=0$ the statement holds, since $\eta D^{0}\left(\frac{1}{\eta}\right)=1=S_{\eta}^{0}(1)$ and so $b_{1}\left(\frac{1}{\eta}\right)=1=$ $d_{\eta, 1}$.

- Suppose that for some $n<m$ we have $\frac{1}{\eta} S_{\eta}^{j}(1)=D^{j}\left(\frac{1}{\eta}\right)$ and $d_{\eta, j}=b_{j}\left(\frac{1}{\eta}\right)$ for all $j \leq n$. Similar to (10) it holds that

$$
D^{n}\left(\frac{1}{\eta}\right)=\frac{2^{n}}{\eta}-b_{1}\left(\frac{1}{\eta}\right) 2^{n-1}-\cdots-b_{n}\left(\frac{1}{\eta}\right)=\frac{2^{n}}{\eta}-d_{\eta, 1} 2^{n-1}-\cdots-d_{\eta, n} .
$$

So, $\frac{1}{\eta} S_{\eta}^{n}(1)=D^{n}\left(\frac{1}{\eta}\right)$. We have $d_{\eta, n+1}=1$ if and only if $S_{\eta}^{n}(1) \in\left(\eta-\frac{1}{2}, 1\right]$, which by the previous holds if and only if $D^{n}\left(\frac{1}{\eta}\right) \in\left(1-\frac{1}{2 \eta}, \frac{1}{\eta}\right] \subseteq\left(\frac{1}{2}, 1\right)$. Hence, $d_{\eta, n+1}=1$ if and only if $b_{n+1}\left(\frac{1}{\eta}\right)=1$. On the other hand, $d_{\eta, n+1}=0$ if and only if $S_{\eta}^{n}(1) \in\left(\eta-1, \frac{1}{2}\right]$, which holds if and only if $D^{n}\left(\frac{1}{\eta}\right) \in\left(1-\frac{1}{\eta}, \frac{1}{2 \eta}\right] \subseteq\left(0, \frac{1}{2}\right)$. Hence $d_{\eta, n+1}=0$ if and only if $b_{n+1}\left(\frac{1}{\eta}\right)=0$. This implies that

$$
S_{\eta}^{n+1}(1)=2 S_{\eta}^{n}(1)-d_{\eta, n+1} \eta=\eta\left(2 D^{n}\left(\frac{1}{\eta}\right)-b_{n+1}\left(\frac{1}{\eta}\right)\right)=\eta D^{n+1}\left(\frac{1}{\eta}\right),
$$

which proves the proposition.

Figure 3 shows the regions where matching occurs for $S_{\eta}$ and $D$. The previous proposition immediately implies a third characterisation of the matching index:

$$
m(\eta)=\inf \left\{n \geq 1: D^{n}\left(\frac{1}{\eta}\right) \in\left(\frac{1}{2 \eta}, 1-\frac{1}{2 \eta}\right)\right\}+1
$$


We use this characterisation in the next proposition.

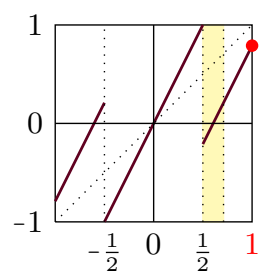

(a) $S_{\eta}$ with the hole $\left(\frac{1}{2}, \eta-\frac{1}{2}\right)$

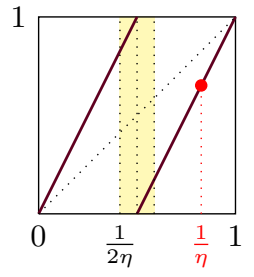

(b) $D$ with the hole $\left(\frac{1}{2 \eta}, 1-\frac{1}{2 \eta}\right)$

Figure 3. There is matching for $S_{\eta}$ if the orbit of 1 enters the yellow region under $S_{\eta}$ in (a) or equivalently if the orbit of $\frac{1}{\eta}$ enters the yellow region under $D$ in (b).

Proposition 2.3. For Lebesgue almost all $\eta \in[1,2]$ it holds that $m(\eta)<\infty$, i.e., $S_{\eta}$ has matching.

Proof. It is enough to consider $\eta \in\left(1, \frac{3}{2}\right)$. Let $k \geq 7$. The ergodicity of $D$ with respect to Lebesgue measure gives that for Lebesgue almost every $x \in(0,1)$ there is an $n \geq 1$, such that $D^{n} x \in\left(\frac{1}{2}-\frac{1}{k}, \frac{1}{2}+\frac{1}{k}\right)$. Since $\eta>\frac{k}{k-2}$ if and only if $\frac{1}{2}+\frac{1}{k}<1-\frac{1}{2 \eta}$, by Proposition 2.2 this means that for almost all $\eta \in\left(\frac{k}{k-2}, \frac{3}{2}\right)$ matching occurs for $S_{\eta}$. Let $A_{k}$ denote the set of all $\eta \in\left(\frac{k}{k-2}, \frac{3}{2}\right)$ such that $S_{\eta}$ does not have matching. Then $A_{k}$ has zero Lebesgue measure and thus also $\bigcup_{k>7} A_{k}$ has zero Lebesgue measure. Since $\bigcup_{k \geq 7} A_{k}$ equals the set of all $\eta \in\left(1, \frac{3}{2}\right)$ such that $S_{\eta}$ does not have matching, this finishes the proof.

Now consider the non-matching set $\mathcal{N}$ of parameters $\eta$ such that $S_{\eta}$ does not have matching:

$$
\begin{aligned}
\mathcal{N} & :=\left\{\eta \in\left(1, \frac{3}{2}\right): m(\eta)=\infty\right\} \\
& =\left\{\eta \in\left(1, \frac{3}{2}\right): D^{n}\left(\frac{1}{\eta}\right) \notin\left(\frac{1}{2 \eta}, 1-\frac{1}{2 \eta}\right) \text { for all } n \geq 1\right\} .
\end{aligned}
$$

One easily checks that $\mathcal{N}=\frac{1}{\Gamma}$, where

$$
\Gamma=\left\{x \in[0,1]: 1-x \leq D^{k}(x) \leq x \text { for all } k \geq 1\right\},
$$

by noting that if $x \in \Gamma$, then $x>2 / 3$, and $D^{k}(x) \notin\left(\frac{x}{2}, 1-\frac{x}{2}\right)$ for all $k$. The set $\Gamma$ was introduced in [AC83, AC01] by Allouche and Cosnard in connection with univoque numbers. In [BCIT13, Lem. 5.5] Bonanno et al. proved that $\Gamma=\Lambda \backslash\{0\}$, 
where

$$
\Lambda=\left\{x \in[0,1]: T^{k}(x) \leq x \text { for all } k \geq 1\right\}
$$

and $T$ denotes the tent map defined by

$$
T:[0,1] \rightarrow[0,1], \quad x \mapsto \min \{2 x, 2-2 x\}
$$

Under an appropriate coding $\Lambda$ can be seen as a representation of all the kneading invariants of unimodal maps. The authors showed, among other things, that $\Lambda$ is closed, uncountable, totally disconnected and has Lebesgue measure 0. In [CT12] it was shown that $\Lambda$ has Hausdorff dimension 1 . Since $\mathcal{N}$ is homeomorphic to $\Lambda \backslash\{0\}$ via the bi-Lipschitz homeomorphism $x \mapsto \frac{1}{x}$ on $[1,2]$, we can use these correspondences to conclude that $\mathcal{N}$ has the following properties:

(i) the set $\mathcal{N}$ has Lebesgue measure 0 ;

(ii) the set $\mathcal{N}$ has Hausdorff dimension 1;

(iii) the set $\mathcal{N}$ is totally disconnected and closed.

Note that these properties also imply that matching occurs Lebesgue almost everywhere.

\section{$\S 3$. Matching intervals for the symmetric doubling maps}

Now that we have established matching on a full measure set, we study the finer matching structure of the maps $S_{\eta}$. In particular, we will identify intervals in $[1,2]$ of parameters such the maps $S_{\eta}$ have the same matching index for all $\eta$ in such an interval. This is achieved by establishing a relation between the symmetric doubling maps and Nakada's $\alpha$-continued fraction maps. In the next subsection we summarise the information on $\alpha$-continued fraction maps that we need here.

\section{$\S 3.1$. Matching for the $\alpha$-continued fraction maps}

The $\alpha$-continued fraction maps were first introduced by Nakada in [Nak81]. They form a one-parameter family of maps $\left\{T_{\alpha}:[\alpha-1, \alpha] \rightarrow[\alpha-1, \alpha]\right\}_{\alpha \in[0,1]}$ defined by $T_{\alpha}(0)=0$ and

$$
T_{\alpha}(x)=\left|\frac{1}{x}\right|-\left\lfloor\left|\frac{1}{x}\right|+1-\alpha\right\rfloor \quad \text { if } x \neq 0 .
$$

Each $T_{\alpha}$ has a unique ergodic absolutely continuous invariant measure $\nu_{\alpha}$. It was later found that the map $\alpha \mapsto h_{\nu_{\alpha}}\left(T_{\alpha}\right)$ has a very intricate structure. Below we mention specifically some results from [CT12, KSS12, BCIT13, CT13] that we use here. 
The matching intervals of the $\alpha$-continued fraction maps can be completely described in terms of continued fraction expansions of rationals in $(0,1]$. Every $x \in(0,1]$ has a continued fraction of the form

$$
x=\frac{1}{a_{1}+\frac{1}{a_{2}+\cdot}}=:\left[0 ; a_{1} a_{2} \cdots\right],
$$

where each $a_{i} \in \mathbb{N}$ and the expansion is infinite if $x \notin \mathbb{Q}$ and finite otherwise. If $x \in \mathbb{Q}$, then there are two possible expansions:

$$
\left[0 ; a_{1} \cdots a_{k}\right]=\left[0 ; a_{1} \cdots a_{k-1}\left(a_{k}-1\right) 1\right], \quad \text { where } a_{k} \geq 2 .
$$

Let $a \in \mathbb{Q} \cap(0,1)$ with continued fraction expansion

$$
a=\left[0 ; a_{1} a_{2} \cdots a_{k}\right]=\left[0 ; a_{1} \cdots a_{k-1}\left(a_{k}-1\right) 1\right] .
$$

From now on, we will always choose to write the expansion of $a$ that has an odd number of digits, so that $a=\left[0 ; a_{1} a_{2} \cdots a_{2 n+1}\right]$. Associate to $a$ the interval $I_{a} \subseteq[0,1]$, given by

$$
I_{a}=\left(a^{-}, a^{+}\right)=\left(\left[0 ;\left(a_{1} \cdots a_{2 n+1}\right)^{\infty}\right],\left[0 ;\left(a_{1} \cdots a_{2 n}\left(a_{2 n+1}-1\right) 1\right)^{\infty}\right]\right)
$$

if $a_{2 n+1} \geq 2$ and

$$
I_{a}=\left(a^{-}, a^{+}\right)=\left(\left[0 ;\left(a_{1} \cdots a_{2 n+1}\right)^{\infty}\right],\left[0 ;\left(a_{1} \cdots a_{2 n-1}\left(a_{2 n}+1\right)\right)^{\infty}\right]\right)
$$

if $a_{2 n+1}=1$, where for any finite string $w_{1} \cdots w_{k}$ we use $\left(w_{1} \cdots w_{k}\right)^{\infty}$ to denote the infinite sequence $w_{1} \cdots w_{k} w_{1} \cdots w_{k} w_{1} \cdots$. The interval $I_{a}=\left(a^{-}, a^{+}\right)$is well defined by (18) and is called the quadratic interval with pseudocentre $a$ in [CT12]. For $a=1$ this procedure does not work and we take $1^{-}=\left[0 ; 1^{\infty}\right]$ and $1^{+}=[0 ; 1]$, so that $I_{1}=(g, 1)$, where $g=\frac{\sqrt{5}-1}{2}$ is the golden mean.

In [CT12, Prop. $2.4 \&$ Lem. 2.6] it was shown that any two quadratic intervals are either disjoint or one is a proper subset of the other. A quadratic interval is then called maximal if it is not properly contained in any other quadratic interval. The maximal quadratic intervals are the matching intervals for the family of maps $\left\{T_{\alpha}\right\}_{\alpha \in[0,1]}$, which in particular implies that the entropy $h_{v_{\alpha}}\left(T_{\alpha}\right)$ is monotone on $I_{a}$. More precisely, if $a=\left[0 ; a_{1} \cdots a_{2 n+1}\right]$ and if we set $N=1+\sum_{j \text { even }} a_{j}$ and $M=-1+\sum_{j \text { odd }} a_{j}$, then in [CT12, Thm. 3.8] it is shown that on $I_{a}$,

$$
h_{\nu_{\alpha}}\left(T_{\alpha}\right) \text { is } \begin{cases}\text { increasing } & \text { if } N-M<0, \\ \text { constant } & \text { if } N-M=0, \\ \text { decreasing } & \text { if } N-M>0 .\end{cases}
$$


In $\left[\mathrm{KSS} 12\right.$, Sect. 10] the authors showed that $\alpha \mapsto h_{\nu_{\alpha}}\left(T_{\alpha}\right)$ is constant on $\left[g^{2}, g\right]$ and their first open question asks whether this is a maximal value that is attained only on this interval. This question remained unanswered for several years and during the writing of this article, but very recently Nakada presented a proof of the claim that this is indeed the maximal interval on which the maximal value of $h_{\nu_{\alpha}}\left(T_{\alpha}\right)$ is attained $([\mathrm{Nak}])$.

The results from [CT12, Prop. $2.13 \&$ Lem. 4.4] characterise the maximal quadratic intervals. Let $<_{g}$ denote the ordering on $\mathbb{N}^{\mathbb{N}}$ given by $\left(c_{n}\right)_{n \geq 1}<_{g}\left(d_{n}\right)_{n \geq 1}$ if and only if $(-1)^{n} c_{n}<(-1)^{n} d_{n}$ for the first index $n$ such that $c_{n} \neq d_{n}$. Then for $x=\left[0 ; c_{1} c_{2} \cdots\right]$ and $y=\left[0 ; d_{1} d_{2} \cdots\right]$ we have

$$
x<y \quad \Leftrightarrow \quad\left(c_{n}\right)_{n \geq 1}<_{g}\left(d_{n}\right)_{n \geq 1} .
$$

The above ordering also applies to finite strings of the same length. Since $a=$ $\left[0 ; a_{1} \cdots a_{2 n+1}\right]$ is assumed to have an odd length, the characterisation of the maximal quadratic intervals from [CT12] reduces to the following: $I_{a}$ is maximal if and only if

$$
a_{1} \cdots a_{2 n+1}<_{g} a_{i+1} \cdots a_{2 n+1} a_{1} \cdots a_{i} \text { for all } i=1, \ldots, 2 n .
$$

Define the binary valuation function $v:\{-1,0,1\}^{\mathbb{N}} \rightarrow \mathbb{R}$ by

$$
v\left(\left(b_{n}\right)_{n \geq 1}\right):=\sum_{n \geq 1} \frac{b_{n}}{2^{n}} .
$$

Let $\{0,1\}^{*}$ be the set of all finite strings of digits in $\{0,1\}$, called words, and use $\epsilon$ to denote the empty word. For any word $w \in\{0,1\}^{*}$ we put $v(w):=v\left(w 0^{\infty}\right)$. Define a function $\varphi:[0,1] \rightarrow[0,1]$ by setting for $x=\left[0 ; a_{1} a_{2} a_{3} \cdots\right]$, that

$$
\varphi(x)=v(\underbrace{11 \cdots 1}_{a_{1}} \underbrace{00 \cdots 0}_{a_{2}} \underbrace{11 \cdots 1}_{a_{3}} \cdots) .
$$

In [BCIT13, Thm. 1.1] the authors proved that $\varphi$ is an orientation-reversing homeomorphism. Moreover, for the bifurcation set

$$
\mathcal{E}:=(0,1) \backslash \bigcup_{\substack{a \in \mathbb{Q} \cap(0,1): \\ I_{a} \text { maximal }}} I_{a}
$$

of parameters $\alpha$ that are not contained in any maximal quadratic interval it was proved in [BCIT13] that $\varphi(\mathcal{E})=\Lambda$, where $\Lambda$ is the set from (15).

\section{$\S 3.2$. Identifying matching intervals and primitive words}

In this section we identify all matching intervals for the symmetric doubling maps by linking them to the maximal quadratic intervals of $\alpha$-continued fraction maps. 
Note that for any $a=\left[0 ; a_{1} \cdots a_{2 n+1}\right] \in \mathbb{Q} \cap(0,1)$ we have

$$
\begin{aligned}
& \varphi\left(a^{-}\right)=v\left(\left(1^{a_{1}} 0^{a_{2}} \cdots 1^{a_{2 n+1}} 0^{a_{1}} 1^{a_{2}} \cdots 0^{a_{2 n+1}}\right)^{\infty}\right), \\
& \varphi\left(a^{+}\right)= \begin{cases}v\left(\left(1^{a_{1}} 0^{a_{2}} \cdots 1^{a_{2 n+1}-1} 0\right)^{\infty}\right) & \text { if } a_{2 n+1} \geq 2, \\
v\left(\left(1^{a_{1}} 0^{a_{2}} \cdots 0^{a_{2 n}+1}\right)^{\infty}\right) & \text { if } a_{2 n+1}=1 .\end{cases}
\end{aligned}
$$

To $a$ we assign the word $w(a) \in\{0,1\}^{*}$ given by

$$
w(a)=1^{a_{1}} 0^{a_{2}} \cdots 1^{a_{2 n+1}} .
$$

Proposition 3.1. For any $a=\left[0 ; a_{1} \cdots a_{2 n+1}\right] \in \mathbb{Q} \cap(0,1)$ the interval $\varphi\left(I_{a}\right)$ is given by

$$
\varphi\left(I_{a}\right)=\left(\varphi\left(a^{+}\right), \varphi\left(a^{-}\right)\right)=\left(\frac{2^{m} \varphi(a)-1}{2^{m}-1}, \frac{2^{m} \varphi(a)+1}{2^{m}+1}\right),
$$

where $m=\sum_{j=1}^{2 n+1} a_{j}$ is the sum of the continued fraction digits of $a$.

Proof. Write $w(a)=w_{1} \cdots w_{m}$. Since $a^{-}=\left[0 ;\left(a_{1} \cdots a_{2 n+1}\right)^{\infty}\right]$, we have

$$
\begin{aligned}
\varphi\left(a^{-}\right) & =v\left(\left(1^{a_{1}} 0^{a_{2}} \cdots 1^{a_{2 n+1}} 0^{a_{1}} 1^{a_{2}} \cdots 1^{a_{2 n+1}}\right)^{\infty}\right) \\
& =v\left(\left(w_{1} \cdots w_{m}\left(1-w_{1}\right) \cdots\left(1-w_{m}\right)\right)^{\infty}\right) .
\end{aligned}
$$

From the binary expansion of $\varphi\left(a^{-}\right)$, we see that $\varphi\left(a^{-}\right)$is the fixed point of the map $D^{2 m}$ corresponding to the digits $w_{1} \cdots w_{m}\left(1-w_{1}\right) \cdots\left(1-w_{m}\right)$, so that $\varphi\left(a^{-}\right)$ is the unique solution to the equation

$$
\begin{aligned}
x=D^{2 m}(x) & =2^{2 m} x-w_{1} 2^{2 m-1}-\cdots-2^{m} w_{m}-2^{m-1}\left(1-w_{1}\right)-\cdots-\left(1-w_{m}\right) \\
& =2^{2 m} x-2^{m} \varphi(a)\left(2^{m}-1\right)-\left(2^{m}-1\right) .
\end{aligned}
$$

Hence,

$$
\varphi\left(a^{-}\right)=\frac{2^{m} \varphi(a)+1}{2^{m}+1} .
$$

A similar calculation shows that

$$
\varphi\left(a^{+}\right)=v\left(\left(w_{1} \cdots w_{m-1} 0\right)^{\infty}\right)=\frac{2^{m} \varphi(a)-1}{2^{m}-1},
$$

since it is the fixed point of the map $D^{m}$ for the branch corresponding to the digits $w_{1} \cdots w_{m-1} 0$. The statement then follows from the fact that $\varphi$ is an orientationreversing homeomorphism.

Recall that $I_{1}=(g, 1)$, where $g$ is the golden mean and note that $\varphi(1)=\frac{1}{2}$ and

$$
\varphi(g)=\sum_{k \geq 1} \frac{1}{2^{2 k-1}}=\frac{2}{3} .
$$


Hence, $\frac{1}{\varphi\left(I_{1}\right)}=\left(\frac{3}{2}, 2\right)$. We know that for each $\eta \in\left(\frac{3}{2}, 2\right)$ the map $S_{\eta}$ has matching after one step; the interval $\frac{1}{\varphi\left(I_{1}\right)}=\left(\frac{3}{2}, 2\right)$ is a matching interval. The next theorem says that in fact each maximal quadratic interval is mapped to a matching interval for the family of symmetric doubling maps by the map $x \mapsto \frac{1}{\varphi(x)}$.

Theorem 3.1. Let $a=\left[0 ; a_{1} \cdots a_{2 n+1}\right] \in \mathbb{Q} \cap(0,1)$ and let $m=\sum_{j=1}^{2 n+1} a_{j}$. Assume that $I_{a}$ is a maximal quadratic interval. Then any $\eta \in \frac{1}{\varphi\left(I_{a}\right)}$ has $m(\eta)=m$ and

$$
d_{\eta, 1}(1) \cdots d_{\eta, m-1}(1)=b_{1}\left(\frac{1}{\eta}\right) \cdots b_{m-1}\left(\frac{1}{\eta}\right)=w_{1}(a) \cdots w_{m-1}(a) .
$$

Proof. Assume that $a_{2 n+1}=1$. The proof for the other case is similar. Write $w(a)=w_{1} \cdots w_{m}$. From Proposition 3.1 we know that

$$
\begin{aligned}
\varphi\left(a^{-}\right) & =v\left(\left(w_{1} \cdots w_{m}\left(1-w_{1}\right) \cdots\left(1-w_{m}\right)\right)^{\infty}\right) \\
\text { and } \quad \varphi\left(a^{+}\right) & =v\left(\left(w_{1} \cdots w_{m-1} 0\right)^{\infty}\right) .
\end{aligned}
$$

Since both $\varphi\left(a^{-}\right)$and $\varphi\left(a^{+}\right)$have a purely periodic binary expansion, their binary expansion is unique. Recall the definitions of $\mathcal{N}$ and $\Lambda$ from (13) and (15) and the fact that $\mathcal{N}=\frac{1}{\Lambda \backslash\{0\}}$. By $(21)$ and the fact that $\varphi(\mathcal{E})=\Lambda$ we have $\frac{1}{\varphi\left(a^{-}\right)}, \frac{1}{\varphi\left(a^{+}\right)} \in \mathcal{N}$. So by Proposition 2.2, $D^{k}\left(\varphi\left(a^{-}\right)\right) \notin\left(\frac{1}{2} \varphi\left(a^{-}\right), 1-\frac{1}{2} \varphi\left(a^{-}\right)\right)$for any $k \geq 0$ and similarly, $D^{k}\left(\varphi\left(a^{+}\right)\right) \notin\left(\frac{1}{2} \varphi\left(a^{+}\right), 1-\frac{1}{2} \varphi\left(a^{+}\right)\right)$for any $k \geq 0$. Note that

$$
\left(\frac{1}{2} \varphi\left(a^{+}\right), 1-\frac{1}{2} \varphi\left(a^{+}\right)\right) \subseteq\left(\frac{1}{2} \varphi\left(a^{-}\right), 1-\frac{1}{2} \varphi\left(a^{-}\right)\right) .
$$

Since the first $m-1$ binary digits of $\varphi\left(a^{-}\right)$and $\varphi\left(a^{+}\right)$coincide, i.e., since

$$
b_{1}\left(\varphi\left(a^{-}\right)\right) \cdots b_{m-1}\left(\varphi\left(a^{-}\right)\right)=b_{1}\left(\varphi\left(a^{+}\right)\right) \cdots b_{m-1}\left(\varphi\left(a^{+}\right)\right)
$$

we have for any $0 \leq k \leq m-2$ that the points $D^{k}\left(\varphi\left(a^{-}\right)\right)$and $D^{k}\left(\varphi\left(a^{+}\right)\right)$either both lie to the left of $\frac{1}{2}$ or both lie to right of $\frac{1}{2}$. Hence, for these values of $k$ the set $D^{k}\left(\varphi\left(I_{a}\right)\right)$ is an interval and

$$
\lambda\left(D^{k}\left(\varphi\left(I_{a}\right)\right)\right)=\lambda\left(\left(D^{k}\left(\varphi\left(a^{-}\right)\right), D^{k}\left(\varphi\left(a^{+}\right)\right)\right)\right)=2^{k} \lambda\left(\varphi\left(I_{a}\right)\right),
$$

where $\lambda$ denotes the one-dimensional Lebesgue measure. For any $x \in \varphi\left(I_{a}\right)$ and $0 \leq k \leq m-2$ we have $D^{k}(x) \in D^{k}\left(\varphi\left(I_{a}\right)\right)$. If it holds that $D^{k}\left(\varphi\left(a^{-}\right)\right) \geq 1-$ $\frac{1}{2} \varphi\left(a^{-}\right)$, then it follows from $(24)$ that $D^{k}(x) \geq 1-\frac{1}{2} x$. If on the other hand $D^{k}\left(\varphi\left(a^{-}\right)\right)<\frac{1}{2} \varphi\left(a^{-}\right)$, then combining that $D^{k}\left(\varphi\left(a^{+}\right)\right)<\frac{1}{2} \varphi\left(a^{+}\right)$and (24), we can also deduce that $D^{k}(x) \leq \frac{1}{2} x$. So $D^{k}(x) \notin\left(\frac{1}{2} x, 1-\frac{1}{2} x\right)$ for any $0 \leq k \leq m-2$.

Next we consider the $(m-1)$ st iteration. By the periodicity and the form of the binary expansions of $\varphi\left(a^{-}\right)$and $\varphi\left(a^{+}\right)$we have

$$
\varphi\left(a^{+}\right)=D^{m}\left(\varphi\left(a^{+}\right)\right)=2 D^{m-1}\left(\varphi\left(a^{+}\right)\right),
$$


so $D^{m-1}\left(\varphi\left(a^{+}\right)\right)=\frac{1}{2} \varphi\left(a^{+}\right)$and similarly,

$$
1-\varphi\left(a^{-}\right)=D^{m}\left(\varphi\left(a^{-}\right)\right)=2 D^{m-1}\left(\varphi\left(a^{-}\right)\right)-1,
$$

so $D^{m-1}\left(\varphi\left(a^{-}\right)\right)=1-\frac{1}{2} \varphi\left(a^{-}\right)$. Hence, $D^{m-1}\left(\varphi\left(I_{a}\right)\right)=\left(\frac{1}{2} \varphi\left(a^{+}\right), 1-\frac{1}{2} \varphi\left(a^{-}\right)\right)$. Since $1-\frac{1}{2} x>1-\frac{1}{2} \varphi\left(a^{-}\right)$we obviously have $D^{m-1}(x)<1-\frac{1}{2} x$. The fact that $D^{m-1}(x)>\frac{1}{2} x$ follows since $\lambda\left(D^{m-1}\left(\varphi\left(I_{a}\right)\right)\right)=2^{m-1} \lambda\left(\varphi\left(I_{a}\right)\right)$.

From the previous theorem we get a complete description of the matching behaviour of $\left\{S_{\eta}\right\}_{\eta \in[1,2]}$ in the sense of Theorem A.

Proof of Theorem A. First note that if $\eta$ is not in the image of any maximal quadratic interval, then $\varphi^{-1}\left(\frac{1}{\eta}\right) \in \mathcal{E}$, where $\mathcal{E}$ is the bifurcation set from (21). Since $\mathcal{N}=\frac{1}{\varphi(\mathcal{E}) \backslash\{0\}}$, this means that $\eta \in \mathcal{N}$ and $S_{\eta}$ does not have matching.

From Theorem 3.1 we know that if $\eta \in \frac{1}{\varphi\left(I_{a}\right)}$ for some maximal quadratic interval $I_{a}$, then $S_{\eta}$ has matching and the matching index $m$ is given by the sum of the continued fraction digits of $a$. Moreover, the first part of the signed binary expansion of 1 is equal to $w_{1}(a) \cdots w_{m}(a)$ for any $\eta \in \frac{1}{\varphi\left(I_{a}\right)}$. If, on the other hand, $\eta \notin \frac{1}{\varphi\left(I_{a}\right)} \cup \mathcal{N}$, then there is another $a^{\prime} \in \mathbb{Q} \cap(0,1]$ with $I_{a^{\prime}}$ maximal and $\eta \in \frac{1}{\varphi\left(I_{a^{\prime}}\right)}$. This automatically implies that either $m(\eta) \neq m$ or

$$
d_{\eta, 1}(1) \cdots d_{\eta, m}(1) \neq w_{1}(a) \cdots w_{m}(a) .
$$

In other words, Theorem 3.1 says that the matching intervals for the family $\left\{S_{\eta}\right\}_{\eta \in(1,2]}$ are precisely the images of the matching intervals for the family $\left\{T_{\alpha}\right\}_{\alpha \in(0,1]}$ under the map $x \mapsto \frac{1}{\varphi(x)}$.

Remark 3.1. The map $x \mapsto \frac{1}{\varphi(x)}$ does not give an isomorphism between the transformations $T_{\alpha}$ and $S_{\frac{1}{\varphi(\alpha)}}$, since that would imply that these maps have the same measure-theoretical entropy and this is in general not the case. The measuretheoretical entropy of $S_{\eta}$ is $\log 2$ for any parameter $\eta$, while there are several articles devoted to a detailed description of the dependence of the measure-theoretic entropy of $T_{\alpha}$ on the parameter $\alpha$, such as [NN08, CT12, KSS12, BCIT13, CT13].

From Theorem 3.1 we see that the matching intervals for $\left\{S_{\eta}\right\}_{\eta \in(1,2]}$ are indexed by the initial part of the digit sequences $d_{\eta}$ up to the matching index $m=m(\eta)$ for the values of $\eta$ that are in the matching interval. We now characterise the initial words in $\{0,1\}^{*}$ that correspond to the matching intervals. Define the function $\psi:\{0,1\}^{*} \backslash\{\epsilon, 0,1\} \rightarrow\{0,1\}^{*}$ by

$$
\psi(w)=\psi\left(w_{1} \cdots w_{m}\right)=w_{1} \cdots w_{m}\left(1-w_{1}\right)\left(1-w_{2}\right) \cdots\left(1-w_{m-1}\right) 1 .
$$


Let $\sigma$ denote the left shift on sequences as before and let $\prec$ denote the lexicographical ordering on $\{0,1\}^{\mathbb{N}}$ and $\{0,1\}^{*}$ where we compare two words of unequal length by adding 0 s at the end.

Definition 3.1. A word $w=w_{1} \cdots w_{m} \in\{0,1\}^{*}$ is called primitive if all the following hold:

(i) $w_{1}=w_{2}=w_{m}=1$;

(ii) $\sigma^{n}\left(\left(w_{1} \cdots w_{m}\right)^{\infty}\right) \preceq\left(w_{1} \cdots w_{m}\right)^{\infty}$ for any $n \geq 0$;

(iii) there is no word $u \in\{0,1\}^{*}$ such that $u \prec w \prec \psi(u)$.

The conditions in the definition follow quite naturally from the dynamics of $S_{\eta}$, while $w_{\ell}=d_{\eta, \ell}(1)=1$ for $\ell=1,2$ is necessary since $\eta \in\left(1, \frac{3}{2}\right)$ and $w_{m}=d_{\eta, m}(1)=1$ since the last digit before matching is a 1 . Condition (ii) is the usual restriction given by the dynamics of the system. In fact, any digit sequence $\left(d_{\eta, n}(x)\right)_{n \geq 1}$ produced by the map $S_{\eta}$ will satisfy the following lexicographical condition: for any $k \geq 0$,

$$
\sigma^{k}\left(d_{\eta, n}(x)\right)_{n \geq 1} \preceq\left(d_{\eta, n}(1)\right)_{n \geq 1} .
$$

Condition (iii) is the actual condition specifying which words correspond to a matching interval. It guarantees that matching occurs exactly at time $m$ and not before.

The notion of primitivity is related, in fact equivalent, to the notion of admissibility which is central in the study of unique expansions (see Remark 3.2 below).

Definition 3.2. A word $w=w_{1} \cdots w_{m} \in\{0,1\}^{*}$ is called admissible if $m \geq 2$ and

$$
\begin{aligned}
\left(1-w_{1}\right) \cdots\left(1-w_{m-k}\right) 0^{\infty} & \prec w_{k+1} \cdots w_{m} 0^{\infty} \\
& \preceq w_{1} \cdots w_{m-k} 0^{\infty} \quad \text { for all } 0 \leq k \leq m-1 .
\end{aligned}
$$

In [Kon], Kong proved the equivalence of the above two notions. We state his result in the following proposition. His proof can be found in the Appendix.

Proposition 3.2. A word $w \in\{0,1\}^{*}$ is primitive if and only if it is admissible.

Recall the definition of $w(a)$ from (23) and associate similarly to each primitive $w \in\{0,1\}^{*}$ a rational number $a \in \mathbb{Q} \cap(0,1)$ by setting

$$
a(w)=\left[0 ; a_{1} \cdots a_{2 n+1}\right] \quad \text { if } w=1^{a_{1}} 0^{a_{2}} \cdots 1^{a_{2 n+1}} .
$$

Using the fact that primitivity is equivalent to admissibility, we get the following result linking maximal quadratic intervals and primitive words. 
Proposition 3.3. Let $w \in\{0,1\}^{*}$ and let $a \in \mathbb{Q} \cap(0,1)$. If $w$ is a primitive word, then $I_{a(w)}$ is a maximal quadratic interval and if $I_{a}$ is a maximal quadratic interval, then $w(a)$ is primitive.

Proof. First let $a=\left[0 ; a_{1} \cdots a_{2 n+1}\right] \in \mathbb{Q} \cap(0,1)$ be given and set $m=a_{1}+\cdots+$ $a_{2 n+1}$. Write $w(a)=w_{1} \cdots w_{m}$. Suppose $I_{a}$ is maximal, so that (19) holds. For any $k$,

$$
w_{k+1} \cdots w_{m}=1^{p} 0^{a_{2 \ell}} \cdots 1^{a_{2 n+1}}, \quad \text { or } w_{k+1} \cdots a_{m}=0^{p} 1^{a_{2 \ell+1}} \cdots 1^{w_{2 n+1}}
$$

for some $p$. In both cases, it follows directly from (19) that

$$
w_{k+1} \cdots w_{m} 0^{\infty} \preceq w_{1} \cdots w_{m-k} 0^{\infty} \quad \text { for all } 0 \leq k \leq m-1 .
$$

So we need only to show that $\left(1-w_{1}\right) \cdots\left(1-w_{m-k}\right) 0^{\infty} \prec w_{k+1} \cdots w_{m} 0^{\infty}$, and for this it suffices to consider the case $w_{k+1} \cdots w_{m}=0^{p} 1^{a_{2 \ell+1}} \cdots 1^{a_{2 n+1}}$. Now $p \leq a_{2 \ell}$ and $a_{2 \ell} \leq a_{1}$. If $p<a_{1}$, then the desired strict inequality holds and we are done. Assume $p=a_{1}$, then $a_{2 \ell}=a_{1}$, and $w_{k+1} \cdots w_{m}=0^{a_{2 \ell}} 1^{a_{2 \ell+1}} \cdots 1^{a_{2 n+1}}$. Assume for the sake of getting a contradiction that $w_{k+1} \cdots w_{m} \preceq\left(1-w_{1}\right) \cdots\left(1-w_{m-k}\right)$. From (19) this implies $w_{k+1} \cdots w_{m}=\left(1-w_{1}\right) \cdots\left(1-w_{m-k}\right)$, and hence $a_{2 \ell} \cdots a_{2 n+1}=$ $a_{1} \cdots a_{2 n-2 \ell+2}$. By repeatedly applying this, we see that there exist integers $r$ and $s \leq 2 \ell-1$ such that

$$
a_{1} \cdots a_{2 n+1}=\left(a_{1} \cdots a_{2 \ell-1}\right)^{r} a_{1} \cdots a_{s}
$$

and

$$
a_{2 \ell} \cdots a_{2 n+1} a_{1} \cdots a_{2 \ell-1}=\left(a_{1} \cdots a_{2 \ell-1}\right)^{r-1} a_{1} \cdots a_{s}\left(a_{1} \cdots a_{2 \ell-1}\right) .
$$

Since

$$
a_{1} \cdots a_{2 n+1}<_{g} a_{2 \ell} \cdots a_{2 n+1} a_{1} \cdots a_{2 \ell-1},
$$

we see that they differ in their last block of length $2 \ell-1$, which occurs at the odd position $2 n-2 \ell+3$. This implies that $a_{s+1} \cdots a_{2 \ell-1} a_{1} \cdots a_{s}<_{g} a_{1} \cdots a_{2 \ell-1}$. However, this contradicts the inequality $a_{1} \cdots a_{2 n+1}<_{g} a_{2 n-2 \ell+3} \cdots a_{2 n+1} a_{1} \cdots$ $a_{2 n-2 \ell+2}$. Hence, $\left(1-w_{1}\right) \cdots\left(1-w_{m-k}\right) 0^{\infty} \prec w_{k+1} \cdots w_{m} 0^{\infty}$, and $w(a)$ is admissible. Then $w(a)$ is primitive by Proposition 3.2.

Now let $w=w_{1} \cdots w_{m}=1^{a_{1}} 0^{a_{2}} \cdots 1^{a_{2 n+1}} \in\{0,1\}^{*}$ be a primitive word. By Proposition 3.2 then $w$ is admissible. To prove that $I_{a(w)}$ is maximal, we verify (19) by considering strings starting with even and odd indices separately.

Let $k=a_{1}+\cdots+a_{2 \ell-1}$. By admissibility we have $\left(1-w_{1}\right) \cdots\left(1-w_{m-k}\right) \prec$ $0^{a_{2 \ell}} \cdots 1^{a_{2 n+1}}$, so there is a least $j \leq 2 n-2 \ell+1$ such that $a_{j} \neq a_{2 \ell+j-1}$. Admissibility also implies that $a_{1} \cdots a_{j}<_{g} a_{\ell} \cdots a_{2 \ell+j-1}$ and hence $a_{1} \cdots a_{2 n+1}<_{g}$ $a_{2 \ell} \cdots a_{2 n+1} a_{1} \cdots a_{2 \ell-1}$ as required. 
We consider now the case $k=a_{1}+\cdots+a_{2 \ell}$. By admissibility,

$$
w_{k+1} \cdots w_{m}=1^{a_{2 \ell+1}} 0^{a_{2 \ell+2}} \cdots 1^{a_{2 n+1}} \preceq w_{1} \cdots w_{m-k} .
$$

If there exists a $1 \leq j \leq 2 n-2 \ell$ such that $a_{j} \neq a_{2 \ell+j}$, then admissibility would imply that $a_{1} \cdots a_{2 n-2 \ell}<_{g} a_{2 \ell} \cdots a_{2 n}$, and hence $a_{1} \cdots a_{2 n+1}<_{g} a_{2 \ell+1} \cdots a_{2 n+1} a_{1} \cdots a_{2 \ell}$. So assume that $a_{j}=a_{2 \ell+j}$ for all $1 \leq j \leq 2 n-2 \ell$. By admissibility, we see that $w_{1} \cdots w_{m-k}=1^{a_{1}} \cdots 0^{a_{2 n-2 \ell}} 1^{t}$ with $t=a_{2 n+1} \leq a_{2 n-2 \ell+1}$. If $a_{2 n+1}<a_{2 n-2 \ell+1}$, then $a_{1} \cdots a_{2 n-2 \ell+1}<_{g} a_{2 \ell} \cdots a_{2 n+1}$ and hence $a_{1} \cdots a_{2 n+1}<_{g} a_{2 \ell+1} \cdots a_{2 n+1} a_{1} \cdots$ $a_{2 \ell}$. We consider now the case $a_{2 n+1}=a_{2 n-2 \ell+1}$. Using the equality $a_{2 \ell+1} \cdots a_{2 n+1}$ $=a_{1} \cdots a_{2 n-2 \ell+1}$ repeatedly, we can write

and

$$
a_{1} \cdots a_{2 n+1}=\left(a_{1} \cdots a_{2 \ell}\right)^{r} a_{1} \cdots a_{s}
$$

$$
a_{2 \ell+1} \cdots a_{2 n+1} a_{1} \cdots a_{2 \ell}=\left(a_{1} \cdots a_{2 \ell}\right)^{r-1} a_{1} \cdots a_{s}\left(a_{1} \cdots a_{2 \ell}\right)
$$

with $s \leq 2 \ell$. We see that they differ in their last block of length $2 \ell$, which occurs at the even position $2 n-2 \ell+2$. Since by admissibility

$\left(1-w_{1}\right) \cdots\left(1-w_{k}\right)=0^{a_{1}} 1^{a_{2}} \cdots 1^{a_{2 \ell}} \prec w_{m-k} \cdots w_{m}=0^{a_{2 n-2 \ell+2}} 1^{a_{2 n-2 \ell+3}} \cdots 1^{a_{2 n+1}}$, this would imply that

and hence

$$
a_{1} \cdots a_{2 \ell}<_{g} a_{s+1} \cdots a_{2 n-2 \ell+2} \cdots a_{2 n+1}=a_{2 \ell} a_{1} \cdots a_{s}
$$

$$
a_{1} \cdots a_{2 n+1}=a_{1} \cdots a_{2 n-2 \ell+1} a_{2 n-2 \ell+2} \cdots a_{2 n+1}<_{g} a_{2 \ell+1} \cdots a_{2 n+1} a_{1} \cdots a_{2 \ell}
$$

as required. Therefore, $I_{a}$ is maximal.

So, every matching interval for $\left\{S_{\eta}\right\}_{\eta \in[1,2]}$ is coded by a primitive word. Next we show that the matching intervals exhibit a period doubling behaviour that has already been observed numerically for the $\alpha$-continued fraction maps in [CMPT10, Sect. 4.2] and investigated further in [BCIT13].

\section{§3.3. Cascades of matching intervals and non-matching parameters}

In the next two propositions we prove that attached to each matching interval we find a whole cascade of matching intervals separated by elements from the nonmatching set $\mathcal{N}$ for which a Markov partition exists and with an accumulation point that is a transcendental element of $\mathcal{N}$. For a primitive word $w$ let $J_{w}=$ $\frac{1}{\varphi\left(I_{a(w)}\right)}$ be a matching interval and set

$$
L(w)=\frac{1}{\varphi\left(a^{-}(w)\right)} \quad \text { and } \quad R(w)=\frac{1}{\varphi\left(a^{+}(w)\right)},
$$

so that $J_{w}=(L(w), R(w))$. Recall the definition of $\psi$ from (25). 
Proposition 3.4. Let $w=w_{1} \cdots w_{m}$ be a primitive word. Then $L(w)=R(\psi(w))$.

Proof. By (22) and Proposition 3.1, $L(w)=\frac{2^{m}+1}{2^{m} v(w)+1}$ and $R(\psi(w))=\frac{2^{2 m}-1}{2^{2 m} v(\psi(w))-1}$. Note that

$$
\begin{aligned}
2^{2 m} v(\psi(w))= & w_{1} 2^{2 m-1}+\cdots+w_{m-1} 2^{m+1}+2^{m}+\left(1-w_{1}\right) 2^{m-1} \\
& +\cdots+\left(1-w_{m-1}\right) 2+1 \\
= & 2^{m}\left(2^{m} v(w)\right)+2^{m}-2^{m} v(w)=2^{m}\left(2^{m} v(w)+1\right)-2^{m} v(w) .
\end{aligned}
$$

This implies

$$
2^{2 m} v(\psi(w))-1=2^{m}\left(2^{m} v(w)+1\right)-\left(2^{m} v(w)+1\right)=\left(2^{m}-1\right)\left(2^{m} v(w)+1\right) .
$$

Hence,

$$
\frac{2^{2 m}-1}{2^{2 m} v(\psi(w))-1}=\frac{\left(2^{m}+1\right)\left(2^{m}-1\right)}{\left(2^{m}-1\right)\left(2^{m} v(w)+1\right)}=\frac{2^{m}+1}{2^{m} v(w)+1} .
$$

It is easy to see that the primitivity of a word $w$ implies the primitivity of $\psi(w)$. So, the previous proposition implies that attached to each matching interval is a cascade of matching intervals corresponding to the words $\psi^{n}(w)$. Set $\underline{w}:=\lim _{n \rightarrow \infty} \psi^{n}(w)$. Note that $\underline{w}$ does not depend on where in the cascade we start. Write $p_{w}=v(\underline{w})$; then

$$
\lim _{n \rightarrow \infty} L\left(\psi^{n}(w)\right)=\lim _{n \rightarrow \infty} \frac{2^{2^{n} m}+1}{2^{2^{n} m} v\left(\psi^{n}(w)\right)+1}=\lim _{n \rightarrow \infty} \frac{1+\frac{1}{2^{2^{n}} m}}{v\left(\psi^{n}(w)\right)+\frac{1}{2^{2^{n} m}}}=\frac{1}{p_{w}} .
$$

Example 3.1. Consider the primitive word 11. Then $J_{11}=\left(\frac{5}{4}, \frac{3}{2}\right)$, so for each $\eta \in J_{11}, S_{\eta}$ has matching after 2 steps. We also have $\psi(11)=1101$ and $J_{1101}=$ $\left(\frac{17}{14}, \frac{5}{4}\right)$. For any $\eta$ in this interval, $S_{\eta}$ has matching after 4 steps. For $\eta=\frac{5}{4}$ we have $1-\eta=-\frac{1}{4}$ and

$$
\begin{array}{ll}
S_{\frac{5}{4}}(1)=\frac{3}{4}, & S_{\frac{5}{4}}^{2}(1)=\frac{1}{4}, \quad S_{\frac{5}{4}}^{3}(1)=\frac{1}{2}, \quad S_{\frac{5}{4}}^{4}(1)=1 . \\
S_{\frac{5}{4}}\left(1-\frac{5}{4}\right)=-\frac{1}{2}, \quad S_{\frac{5}{4}}^{2}\left(-\frac{1}{4}\right)=-1, \quad S_{\frac{5}{4}}^{3}\left(-\frac{1}{4}\right)=-\frac{3}{4}, \quad S_{\frac{5}{4}}^{4}\left(-\frac{1}{4}\right)=-\frac{1}{4},
\end{array}
$$

so there is a Markov partition. The limit $\underline{11}=\lim _{n \rightarrow \infty} \psi^{n}(11)$ is the shifted ThueMorse sequence. Recall that the Thue-Morse substitution is given by

$$
0 \mapsto 01, \quad 1 \mapsto 10
$$

The Thue-Morse sequence is the fixed point of this substitution, which is

$$
t=01101001100101101001011001101001 \cdots,
$$


and the Thue-Morse constant $p^{*}$ is the number that has this sequence as its base 2 expansions, i.e., $p^{*}=\sum_{n \geq 1} \frac{t_{n}}{2^{n}}=0.4124 \ldots$ The limit sequence $\underline{11}$ is the sequence obtained when we shift the Thue-Morse sequence one place to the left, $\underline{11}=\sigma(t)$. For the corresponding constant we get

$$
\lim _{n \rightarrow \infty} L\left(\psi^{n}(11)\right)=\frac{1}{p_{11}}=\frac{1}{2 p^{*}}=1.2122 \ldots,
$$

which is transcendental (see [Dek77]).

The previous example illustrates a general pattern. Let $w=w_{1} \cdots w_{m} \in$ $\{0,1\}^{m}$ be a primitive word and consider the right end point $R(w)$ of the matching interval $J_{w}$. Then $d_{R(w)}=\left(w_{1} \cdots w_{m-1} 0\right)^{\infty}$ by $(22)$ and $R(w) \in \mathcal{N}$. It holds that $S_{R(w)}^{m}(1)=1$ and thus $S_{R(w)}^{m}(1-R(w))=1-R(w)$. In other words, $S_{R(w)}$ has a Markov partition, but no matching. Results from [BCIT13] give us the following proposition.

Proposition 3.5. Let $w \in\{0,1\}^{m}$ be a primitive word. Then $\frac{1}{p_{w}} \in \mathcal{N}$ and $\frac{1}{p_{w}}$ is transcendental.

Proof. Since $\mathcal{N}$ contains exactly those points that are not in any matching interval, we have $\frac{1}{p_{w}}=\lim _{n \rightarrow \infty} L\left(\psi^{n}(w)\right) \in \mathcal{N}$. In [BCIT13] the maps $\tau_{0}$ and $\Delta$ are defined on words $u=u_{1} \cdots u_{n} \in\{0,1\}^{*}$ by $\tau_{0}(u)=v\left(\left(u_{1} \cdots u_{n} 0\right)^{\infty}\right)$ and $\Delta(u)=$ $u_{1} \cdots u_{n} 1\left(1-u_{1}\right) \cdots\left(1-u_{n}\right)$. Then for each $j \geq 1$ they set $\tau_{j}(u)=\tau_{0}\left(\Delta^{j}(u)\right)$ and $\tau_{\infty}(u)=\lim _{j \rightarrow \infty} \tau_{j}(u)$. It is proven in [BCIT13, Prop. 4.7] that if $\tau_{0}(u) \in \Lambda$, then $\tau_{\infty}(u)$ is transcendental. The fact that $\frac{1}{p_{w}}$ is transcendental thus follows from [BCIT13, Prop. 4.7] by observing that $p_{w}=\tau_{\infty}\left(w_{1} \cdots w_{m-1}\right)$.

Remark 3.2. We have already seen that $\mathcal{N}=\frac{1}{\varphi(\mathcal{E}) \backslash\{0\}}$, where $\mathcal{E}$ is the bifurcation set for the family $\left\{T_{\alpha}\right\}_{\alpha \in[0,1]}$ defined in (21). In [BCIT13] the set $\mathcal{E}$ was linked to the real slice of the boundary of the Mandelbrot set, the set $\Lambda$ of codings for the kneading invariants of unimodal maps encoded by $\Lambda$, and the set of univoque bases. So, this article adds a new item to this list of correspondences. The common link between the results from [BCIT13] and our case is the set $\Gamma$ from (14), which was shown by Allouche and Cosnard ([AC83, AC01]) to have connections with univoque numbers. Given a number $1<\beta<2$, one can express any real number $x \in\left[0, \frac{1}{\beta-1}\right]$ as a $\beta$-expansion: $x=\sum_{n \geq 1} \frac{c_{n}}{\beta^{n}}$ for some sequence $\left(c_{n}\right)_{n \geq 1} \in\{0,1\}^{\mathbb{N}}$. Typically a number $x$ has uncountably many different expansions of this form. The number $1<\beta<2$ is called univoque if there is a unique sequence $\left(c_{n}\right)_{n \geq 1} \in\{0,1\}^{\mathbb{N}}$ such that $1=\sum_{n>1} \frac{c_{n}}{\beta^{n}}$, i.e., if 1 has a unique $\beta$-expansion. Let $\mathcal{U}$ denote the set of univoque bases. The properties of $\mathcal{U}$ have been studied by many authors. There exists an equivalent characterisation of univoque numbers in terms of strongly 
admissible sequences, which is mainly due to Parry ([Par60]) (see also [EJK90]) and which is stronger than the notion of admissibility from Definition 3.2.

A sequence $\left(c_{n}\right)_{n \geq 1} \in\{0,1\}^{\mathbb{N}}$ is strongly admissible if and only if

$$
\begin{cases}c_{k+1} c_{k+2} \cdots \prec c_{1} c_{2} \cdots & \text { if } c_{k}=0, \\ c_{k+1} c_{k+2} \cdots \succ c_{1} c_{2} \cdots & \text { if } c_{k}=1,\end{cases}
$$

for all $k$. It is easy to check that strong admissibility is equivalent to

$$
\left(1-c_{1}\right)\left(1-c_{2}\right) \cdots \prec c_{k+1} c_{k+2} \cdots \prec c_{1} c_{2} \cdots
$$

for all $k \geq 1$. In [EJK90] it is proved that a sequence $\left(c_{n}\right)_{n \geq 1}$ is strongly admissible if and only if there is a univoque $\beta>1$ such that $1=\sum_{n \geq 1} \frac{c_{n}}{\beta^{n}}$. In [KL07], the authors studied the topological structure of the set $\mathcal{U}$ as well as its closure $\overline{\mathcal{U}}$. Furthermore, they characterised the sequences that belong to $\overline{\mathcal{U}}$, namely $\left(d_{n}\right)_{n \geq 1} \in$ $\overline{\mathcal{U}}$ if and only if

$$
\left(1-d_{1}\right)\left(1-d_{2}\right) \cdots \prec d_{k+1} d_{k+2} \cdots \preceq d_{1} d_{2} \cdots
$$

for all $k \geq 1$. They also showed that if $\left(d_{n}\right)_{n \geq 1} \in \overline{\mathcal{U}}$, then $\left(d_{n}\right)_{n \geq 1}$ has an arbitrarily long initial block that is admissible in the sense of Definition 3.2. In [AC01] the authors showed that there is a one-to-one correspondence between strong admissible sequences and the points in $\Gamma$ that do not have a periodic binary expansion. In this way the univoque bases are related to a subset of $\mathcal{N}$.

\section{$\S 4$. The continuity of the frequency function}

In this section we consider the unique absolutely continuous invariant measure $\mu_{\eta}$ and the associated frequency function $\eta \mapsto \mu_{\eta}\left(\left[-\frac{1}{2}, \frac{1}{2}\right]\right)$ with the goal of giving an explicit formula for $\mu_{\eta}\left(\left[-\frac{1}{2}, \frac{1}{2}\right]\right)$ on each of the matching intervals $J_{w}$. Recall the expression for the invariant probability density from (8). Suppose that for some $\eta$ we have matching after $m$ steps. Hence, $S_{\eta}^{m}(1)=S_{\eta}^{m}(1-\eta)$ and $S_{\eta}^{m}(\eta-$ 1) $=S_{\eta}^{m}(-1)$. Moreover, before matching we have $S_{\eta}^{n}(1)=S_{\eta}^{n}(1-\eta)+\eta$ and $S_{\eta}^{n}(\eta-1)=S_{\eta}^{n}(-1)+\eta$. Then

$$
f_{\eta}(x)=\frac{1}{C} \sum_{n=0}^{m-1} \frac{1}{2^{n+1}}\left(1_{\left[S_{\eta}^{n}(1-\eta), S_{\eta}^{n}(1)\right)}(x)+1_{\left[S_{\eta}^{n}(-1), S_{\eta}^{n}(\eta-1)\right)}(x)\right),
$$

where $C$ is the normalising constant. Further, $C$ is related to the total measure, 
which is

$$
\begin{aligned}
\mu_{\eta}([-1,1]) & =\frac{1}{C} \int_{-1}^{1} f_{\eta}(x) d x=\frac{2}{C} \sum_{n=0}^{m-1} \frac{1}{2^{n+1}}\left(S_{\eta}^{n}(1)-S_{\eta}^{n}(1-\eta)\right) \\
& =\frac{\eta}{C}\left(\frac{1-\frac{1}{2^{m}}}{1-\frac{1}{2}}\right)=\frac{2 \eta}{C}\left(1-\frac{1}{2^{m}}\right)=1,
\end{aligned}
$$

where we have used that $S_{\eta}^{n}(1)=S_{\eta}^{n}(1-\eta)+\eta$ before matching. Hence,

$$
\frac{1}{C}=\frac{1}{\eta} \frac{2^{m-1}}{2^{m}-1} .
$$

We first prove that the map $\eta \mapsto f_{\eta}$ is continuous.

Theorem 4.1. Let $\bar{\eta} \in[1,2]$ and let $\left(\eta_{k}\right)_{k \geq 1} \subseteq[1,2]$ be a sequence converging to $\bar{\eta}$. Then $f_{\eta_{k}} \rightarrow f_{\bar{\eta}}$ in $L^{1}$, where $f_{\bar{\eta}}=\frac{1}{2}$ in the case $\bar{\eta}=1$.

For the proof we use the Perron-Frobenius operator $\mathcal{L}_{\eta}$ for $S_{\eta}$, which is given by

$$
\left(\mathcal{L}_{\eta} f\right)(x)=\frac{1}{2}\left(f\left(\frac{x}{2}\right)+1_{(\eta-2, \eta-1)}(x) f\left(\frac{x-\eta}{2}\right)+1_{(1-\eta, 2-\eta)}(x) f\left(\frac{x+\eta}{2}\right)\right)
$$

for $f \in L^{1}(\lambda)$, where as before $\lambda$ denotes the one-dimensional Lebesgue measure. For a function $f:[-1,1] \rightarrow \mathbb{R}$, let $\operatorname{Var}(f)$ denote its total variation and use BV to denote the set of functions $f:[-1,1] \rightarrow \mathbb{R}$ of bounded variation, so with $\operatorname{Var}(f)<$ $\infty$. Since $S_{\eta}$ is an expanding interval map with constant slope, it is well known (see for example [BG97, Thm. 5.2.1]) that an absolutely continuous invariant density for $S_{\eta}$ is obtained from the Lebesgue almost everywhere limit of a subsequence of $\left(\frac{1}{n} \sum_{j=0}^{n-1} \mathcal{L}_{\eta}^{j}(1)\right)_{n \geq 1}$.

Proof of Theorem 4.1. First let $\eta \neq \frac{3}{2}$. Let $\mathcal{P}_{\eta}$ be the collection of intervals of monotonicity of $S_{\eta}^{2}$. To be precise, for $\eta \in\left[1, \frac{3}{2}\right)$ we have

$$
\begin{array}{r}
\mathcal{P}_{\eta}=\left\{\left(-1,-\frac{2 \eta+1}{4}\right),\left(-\frac{2 \eta+1}{4},-\frac{1}{2}\right),\left(-\frac{1}{2},-\frac{1}{4}\right),\left(-\frac{1}{4}, \frac{1}{4}\right),\left(\frac{1}{4}, \frac{1}{2}\right),\right. \\
\left.\left(\frac{1}{2}, \frac{2 \eta+1}{4}\right),\left(\frac{2 \eta+1}{4}, 1\right)\right\},
\end{array}
$$

and for $\eta \in\left(\frac{3}{2}, 2\right]$ this becomes

$$
\begin{array}{r}
\mathcal{P}_{\eta}=\left\{\left(-1, \frac{1-2 \eta}{4}\right),\left(\frac{1-2 \eta}{4},-\frac{1}{2}\right),\left(-\frac{1}{2},-\frac{1}{4}\right),\left(-\frac{1}{4}, \frac{1}{4}\right),\left(\frac{1}{4}, \frac{1}{2}\right),\right. \\
\left.\left(\frac{1}{2}, \frac{2 \eta-1}{4}\right),\left(\frac{2 \eta-1}{4}, 1\right)\right\} .
\end{array}
$$


Since $S_{\eta}$ is a piecewise expanding interval map with constant slope it follows from [BG97, Lem. 5.2.1] that for $f \in \mathrm{BV}$,

$$
\begin{aligned}
\operatorname{Var}\left(\mathcal{L}_{\eta} f\right) & \leq \operatorname{Var}(f)+2 \int_{[-1,1]}|f| d \lambda \\
\operatorname{Var}\left(\mathcal{L}_{\eta}^{2} f\right) & \leq \frac{1}{2} \operatorname{Var}(f)+\frac{1}{2 \delta(\eta)} \int_{[-1,1]}|f| d \lambda,
\end{aligned}
$$

where $\delta(\eta)=\min \left\{\lambda(I): I \in \mathcal{P}_{\eta}\right\}=\left|\frac{2 \eta-3}{4}\right|$. For $n \geq 2$, write $n=2 j+i$ with $i \in\{0,1\}$. Then

$$
\begin{aligned}
\operatorname{Var}\left(\mathcal{L}_{\eta}^{n} f\right) & =\operatorname{Var}\left(\mathcal{L}_{S_{\eta}^{2}}^{j} \mathcal{L}_{\eta}^{i} f\right) \leq \frac{1}{2^{j}} \operatorname{Var}\left(\mathcal{L}_{\eta}^{i} f\right)+\sum_{k=0}^{j-1} \frac{1}{2^{k}} \frac{1}{2 \delta(\eta)} \int_{[-1,1]}|f| d \lambda \\
& \leq \frac{1}{2^{j}} \operatorname{Var}(f)+\int_{[-1,1]}|f| d \lambda\left(2+\frac{1}{\delta(\eta)}\right) .
\end{aligned}
$$

Fix some $\bar{\eta} \in(1,2] \backslash\left\{\frac{3}{2}\right\}$ and let $\left(\eta_{k}\right)_{k \geq 1} \subseteq(1,2]$ be a sequence converging to $\bar{\eta}$. Let

$$
0<\varepsilon<\min \left\{\left|\bar{\eta}-\frac{3}{2}\right|, \delta(\bar{\eta})\right\} .
$$

Then for all $\eta \in[\bar{\eta}-\varepsilon, \bar{\eta}+\varepsilon]$, we have $\delta(\eta) \geq \delta(\bar{\eta})-\frac{1}{2} \varepsilon \geq \frac{\delta(\bar{\eta})}{2}$. For $k \geq 1$, define the sequence of functions $f_{k, n}=\frac{1}{n} \sum_{j=0}^{n-1} \mathcal{L}_{\eta_{k}}^{j}(1)$. Recall that since each $S_{\eta_{k}}$ has a unique absolutely continuous invariant measure there is a subsequence of $\left(f_{k, n}\right)$ converging to $f_{\eta_{k}} \lambda$-a.e. For ease of notation we simply assume that $\lim _{n \rightarrow \infty} f_{k, n}=f_{\eta_{k}} \lambda$-a.e. By $(27)$,

$$
\operatorname{Var}\left(f_{k, n}\right) \leq \frac{1}{n} \sum_{j=0}^{n-1} \operatorname{Var}\left(\mathcal{L}_{\eta_{k}}^{j}(1)\right) \leq 4+\frac{2}{\delta\left(\eta_{k}\right)},
$$

so for all $k$ sufficiently large, we have

$$
\operatorname{Var}\left(f_{k, n}\right) \leq 4+\frac{2}{\delta(\bar{\eta})-\frac{1}{2} \varepsilon} \leq 4+\frac{4}{\delta(\bar{\eta})} .
$$

Also,

$$
\begin{aligned}
\sup \left|f_{k, n}\right| & \leq \operatorname{Var}\left(f_{k, n}\right)+\int_{[-1,1]} f_{k, n} d \lambda \\
& \leq \operatorname{Var}\left(f_{k, n}\right)+\frac{1}{n} \sum_{j=0}^{n-1} \int_{[-1,1]} \mathcal{L}_{\eta_{k}}^{j}(1) d \lambda \leq 6+\frac{4}{\delta(\bar{\eta})} .
\end{aligned}
$$

Since both of these bounds are independent of $\eta_{k}$ and $n$, we have $\operatorname{Var}\left(f_{\eta_{k}}\right)$, $\sup \left|f_{\eta_{k}}\right| \leq 6+\frac{4}{\delta(\bar{\eta})}$ for each $k$ large enough. From Helly's theorem it then follows that there is a subsequence $\left(k_{i}\right)$ and an $f_{\infty} \in \mathrm{BV}$ such that $f_{\eta_{k_{i}}} \rightarrow f_{\infty}$ in 
$L^{1}(\lambda)$ and $\lambda$-a.e. and with $\sup \left|f_{\infty}\right|, \operatorname{Var}\left(f_{\infty}\right) \leq 6+\frac{4}{\delta(\bar{\eta})}$. We show that $f_{\infty}=f_{\bar{\eta}}$ by proving that for each Borel set $B \subseteq[-1,1]$ we have

$$
\int_{B} f_{\infty} d \lambda=\int_{S_{\bar{\eta}}^{-1}(B)} f_{\infty} d \lambda
$$

The desired result then follows from the uniqueness of the invariant probability density. First note that $1_{B} \in L^{1}(\lambda)$, so it can be approximated arbitrarily closely by compactly supported $C^{1}$ functions. So instead of (28) we prove that

$$
\left|\int_{[-1,1]}\left(g \circ S_{\bar{\eta}}\right) f_{\infty} d \lambda-\int_{[-1,1]} g f_{\infty} d \lambda\right|=0
$$

for any compactly supported $C^{1}$ function $g$ on $[-1,1]$. (Hence $\|g\|_{\infty}<\infty$.) We split this into three parts:

$$
\begin{aligned}
\left|\int_{[-1,1]}\left(g \circ S_{\bar{\eta}}\right) f_{\infty} d \lambda-\int_{[-1,1]} g f_{\infty} d \lambda\right| \\
\leq\left|\int_{[-1,1]}\left(g \circ S_{\bar{\eta}}\right) f_{\infty} d \lambda-\int_{[-1,1]}\left(g \circ S_{\bar{\eta}}\right) f_{\eta_{k_{i}}} d \lambda\right| \\
\quad+\left|\int_{[-1,1]}\left(g \circ S_{\bar{\eta}}\right) f_{\eta_{k_{i}}} d \lambda-\int_{[-1,1]}\left(g \circ S_{\eta_{k_{i}}}\right) f_{\eta_{k_{i}}} d \lambda\right| \\
\quad+\left|\int_{[-1,1]}\left(g \circ S_{\eta_{k_{i}}}\right) f_{\eta_{k_{i}}} d \lambda-\int_{[-1,1]} g f_{\infty} d \lambda\right| .
\end{aligned}
$$

Then for the first part we have

$$
\begin{aligned}
& \left|\int_{[-1,1]}\left(g \circ S_{\bar{\eta}}\right) f_{\infty} d \lambda-\int_{[-1,1]}\left(g \circ S_{\bar{\eta}}\right) f_{\eta_{k_{i}}} d \lambda\right| \\
& \quad \leq\left|\int_{[-1,1]}\left(\sup _{x \in[-1,1]} g(x)\right)\left(f_{\infty}-f_{\eta_{k_{i}}}\right) d \lambda\right| \\
& \quad \leq\|g\|_{\infty} \int_{[-1,1]}\left|f_{\infty}-f_{\eta_{k_{i}}}\right| d \lambda=\|g\|_{\infty}\left\|f_{\infty}-f_{\eta_{k_{i}}}\right\|_{L^{1}} .
\end{aligned}
$$

For the third part we get

$$
\left|\int_{[-1,1]}\left(g \circ S_{\eta_{k_{i}}}\right) f_{\eta_{k_{i}}} d \lambda-\int_{[-1,1]} g f_{\infty} d \lambda\right| \leq\|g\|_{\infty}\left\|f_{\infty}-f_{\eta_{k_{i}}}\right\|_{L^{1}}
$$


Hence, these two parts converge to 0 as $i \rightarrow \infty$. Now, for the middle part we have

$$
\begin{aligned}
& \left|\int_{[-1,1]}\left(g \circ S_{\bar{\eta}}\right) f_{\eta_{k_{i}}} d \lambda-\int_{[-1,1]}\left(g \circ S_{\eta_{k_{i}}}\right) f_{\eta_{k_{i}}} d \lambda\right| \\
& \quad \leq\left(\sup _{x \in[-1,1]} f_{\eta_{k_{i}}}(x)\right) \int_{[-1,1]}\left|\left(g \circ S_{\bar{\eta}}\right)-\left(g \circ S_{\eta_{k_{i}}}\right)\right| d \lambda \\
& \quad \leq\left(6+\frac{4}{\delta(\bar{\eta})}\right) \int_{[-1,1]}\left|\left(g \circ S_{\bar{\eta}}\right)-\left(g \circ S_{\eta_{k_{i}}}\right)\right| d \lambda .
\end{aligned}
$$

By the dominated convergence theorem and the continuity of $g$, we have

$\lim _{i \rightarrow \infty} \int_{[-1,-1 / 2)}\left|\left(g \circ S_{\bar{\eta}}\right)-\left(g \circ S_{\eta_{k_{i}}}\right)\right| d \lambda=\int_{-1}^{-1 / 2} \lim _{i \rightarrow \infty}\left|g\left(2 x+\eta_{k_{i}}\right)-g(2 x+\eta)\right| d x=0$,

and similarly the integral converges to 0 on $\left[-\frac{1}{2}, \frac{1}{2}\right]$ and $\left(\frac{1}{2}, 1\right]$. Hence, $f_{\infty}=f_{\bar{\eta}}$ Lebesgue a.e. In fact, the proof shows that for each subsequence of $\left(f_{\eta_{k}}\right)$ there is a further subsequence that converges a.e. (and in $L^{1}$ ) to $f_{\bar{\eta}}$. This is equivalent to saying that the sequence $\left(f_{\eta_{k}}\right)$ converges in measure to $f_{\bar{\eta}}$. Since $f_{\eta_{k}} \leq 6+\frac{4}{\delta(\bar{\eta})}$ for any $k$ large enough, this implies that $\left(f_{\eta_{k}}\right)$ is uniformly integrable from a certain $k$ on, so by Vitali's theorem the sequence $\left(f_{\eta_{k}}\right)$ converges in $L^{1}$ to $f_{\bar{\eta}}$.

Let $\left(\eta_{k}\right)$ now be a sequence of parameters converging to 1 . By the above arguments the corresponding densities $f_{\eta_{k}}$ converge in $L^{1}$ to an invariant density $f_{\infty}$ for the map $S_{1}$. By (8) it is clear that for each Borel set $B$ and each $k$ it holds that $\mu_{\eta_{k}}(B)=\mu_{\eta_{k}}(-B)$. Hence the same holds for the invariant measure $\mu_{\infty}$ of $S_{1}$ with density $f_{\infty}$. Since each absolutely continuous invariant measure of $S_{1}$ is a convex combination of Lebesgue measures concentrated on the left and right halves of the interval, this implies that $\mu_{\infty}$ is the normalised Lebesgue measure on $[-1,1]$.

The above proof shows that $\eta \mapsto f_{\eta}$ is continuous at any point $\bar{\eta} \in[1,2] \backslash\left\{\frac{3}{2}\right\}$. For $\frac{3}{2}$, we have by (11) that

$$
\begin{aligned}
& \lim _{\eta \downarrow \frac{3}{2}} \int_{[-1,1]}\left|f_{\eta}-f_{\frac{3}{2}}\right| d \lambda \\
& \quad=\lim _{\eta \downarrow \frac{3}{2}}\left(2 \int_{-1}^{1-\eta}\left(\frac{1}{3}-\frac{1}{2 \eta}\right) d x+2 \int_{1-\eta}^{-\frac{1}{2}}\left(\frac{1}{\eta}-\frac{1}{3}\right) d x+\int_{-\frac{1}{2}}^{\frac{1}{2}}\left(\frac{2}{3}-\frac{1}{\eta}\right) d x\right)=0 .
\end{aligned}
$$

For any $\eta$ in the matching interval $J_{11}=\left(\frac{4}{3}, \frac{3}{2}\right)$ that is close enough to $\frac{3}{2}$ we have

$$
f_{\eta}=\frac{1}{6 \eta}\left(2+1_{(2-2 \eta, 2 \eta-2)}+1_{(\eta-2,2-\eta)}+2 \cdot 1_{(1-\eta, \eta-1)}\right),
$$


which is easily checked by direct computation. Then

$$
\begin{aligned}
\lim _{\eta \uparrow \frac{3}{2}} \int_{[-1,1]}\left|f_{\eta}-f_{\frac{3}{2}}\right| d \lambda=\lim _{\eta \uparrow \frac{3}{2}} & \left(2 \int_{-1}^{2-2 \eta}\left(\frac{1}{3}-\frac{1}{3 \eta}\right) d x+2 \int_{2-2 \eta}^{\eta-2}\left(\frac{1}{2 \eta}-\frac{1}{3}\right) d x\right. \\
& +2 \int_{\eta-2}^{-\frac{1}{2}}\left(\frac{2}{3 \eta}-\frac{1}{3}\right) d x+2 \int_{-\frac{1}{2}}^{1-\eta}\left(\frac{2}{3}-\frac{2}{3 \eta}\right) d x \\
& \left.+\int_{1-\eta}^{\eta-1}\left(\frac{2}{3}-\frac{1}{\eta}\right) d x\right)=0 .
\end{aligned}
$$

This gives the result also for $\eta=\frac{3}{2}$.

Corollary 4.1. The frequency function $\eta \mapsto \mu_{\eta}\left(\left[-\frac{1}{2}, \frac{1}{2}\right]\right)$ is continuous on $[1,2]$.

Proof. This immediately follows from the previous result, since for any $\eta \in[1,2]$ and any sequence $\left\{\eta_{k}\right\}_{k \geq 1} \subseteq[1,2]$ converging to $\eta$, we have

$$
\begin{aligned}
\lim _{k \rightarrow \infty}\left|\mu_{\eta}\left(\left[-\frac{1}{2}, \frac{1}{2}\right]\right)-\mu_{\eta_{k}}\left(\left[-\frac{1}{2}, \frac{1}{2}\right]\right)\right| & \leq \lim _{k \rightarrow \infty} \int_{\left[-\frac{1}{2}, \frac{1}{2}\right]}\left|f_{\eta}-f_{\eta_{k}}\right| d \lambda \\
& \leq \lim _{k \rightarrow \infty} \int_{[-1,1]}\left|f_{\eta}-f_{\eta_{k}}\right| d \lambda=0 .
\end{aligned}
$$

We now give a precise description of the measure of the middle interval $\left[-\frac{1}{2}, \frac{1}{2}\right]$. The fact that before matching $S_{\eta}^{k}(1)=S_{\eta}^{k}(1-\eta)+\eta$ in particular implies that $S_{\eta}^{k}(1)$ will visit only $\left[-\frac{1}{2}, \frac{1}{2}\right]$ and $\left(\frac{1}{2}, 1\right]$ and $S_{\eta}^{k}(1-\eta)$ will visit only $\left[-1,-\frac{1}{2}\right)$ and $\left[-\frac{1}{2}, \frac{1}{2}\right]$. Moreover, $S_{\eta}^{k}(1)$ and $S_{\eta}^{k}(1-\eta)$ will never both be in $\left[-\frac{1}{2}, \frac{1}{2}\right]$. We also know that matching occurs immediately after $S_{\eta}^{k}(1) \in\left(\frac{1}{2}, 1\right]$ and $S_{\eta}^{k}(1-\eta) \in\left[-1,-\frac{1}{2}\right)$. So, up to one step before matching we always have exactly one of the two orbits in $\left[-\frac{1}{2}, \frac{1}{2}\right]$.

Let $w=w_{1} \cdots w_{m}$ be a primitive word and $\eta \in J_{w}$. In order to determine $\mu_{\eta}\left(\left[-\frac{1}{2}, \frac{1}{2}\right]\right)$ we need to describe functions of the form

$$
x \mapsto 1_{\left[S_{\eta}^{k}(1-\eta), S_{\eta}^{k}(1)\right)}(x) 1_{\left[-\frac{1}{2}, \frac{1}{2}\right]}(x) .
$$

Note that for $0 \leq k \leq m-2$ if $w_{k+1}=1$, then

$$
1_{\left[S_{\eta}^{k}(1-\eta), S_{\eta}^{k}(1)\right)}(x) 1_{\left[-\frac{1}{2}, \frac{1}{2}\right]}(x)=1_{\left[S_{\eta}^{k}(1-\eta), \frac{1}{2}\right]}(x)
$$

and if $w_{k+1}=0$, then

$$
1_{\left[S_{\eta}^{k}(1-\eta), S_{\eta}^{k}(1)\right)}(x) 1_{\left[-\frac{1}{2}, \frac{1}{2}\right]}(x)=1_{\left[-\frac{1}{2}, S_{\eta}^{k}(1)\right)}(x) .
$$

Moreover,

$$
1_{\left[S_{\eta}^{m-1}(1-\eta), S_{\eta}^{m-1}(1)\right)}(x) 1_{\left[-\frac{1}{2}, \frac{1}{2}\right]}(x)=1_{\left[-\frac{1}{2}, \frac{1}{2}\right]}(x) .
$$


Due to symmetry, the measure of $\left[-\frac{1}{2}, \frac{1}{2}\right]$ is then given by

$$
\begin{aligned}
\mu_{\eta} & \left(\left[-\frac{1}{2}, \frac{1}{2}\right]\right)=\frac{1}{C} \int_{\left[-\frac{1}{2}, \frac{1}{2}\right]} f_{\eta}(x) d x \\
& =\frac{1}{C} \sum_{\substack{0 \leq k \leq m-2: \\
w_{k+1=1}}} \frac{1}{2^{k}}\left(\frac{1}{2}-S_{\eta}^{k}(1-\eta)\right)+\frac{1}{C} \sum_{\substack{0 \leq k \leq m-2: \\
w_{k+1=0}}} \frac{1}{2^{k}}\left(S_{\eta}^{k}(1)+\frac{1}{2}\right)+\frac{1}{C 2^{m-1}} \\
& =\frac{1}{C}\left(\frac{1}{2^{m-1}}+\sum_{k=0}^{m-2} \frac{1}{2^{k+1}}+\sum_{\substack{0 \leq k \leq m-2: \\
w_{k+1=1}}}\left(\frac{\eta}{2^{k}}-\frac{1}{2^{k}} S_{\eta}^{k}(1)\right)+\sum_{\substack{0 \leq k \leq m-2: \\
w_{k+1=0}}} \frac{1}{2^{k}} S_{\eta}^{k}(1)\right) .
\end{aligned}
$$

Observe that if $m=1$, the above two summations are zero. In this case by (26),

$$
\mu_{\eta}\left(\left[-\frac{1}{2}, \frac{1}{2}\right]\right)=\frac{1}{C}=\frac{1}{\eta}
$$

as we saw before. For $m \geq 2$, we get

$$
\mu_{\eta}\left(\left[-\frac{1}{2}, \frac{1}{2}\right]\right)=\frac{1}{C}\left(1+\sum_{\substack{0 \leq k \leq m-2: \\ w_{k+1=1}}}\left(\frac{\eta}{2^{k}}-\frac{1}{2^{k}} S_{\eta}^{k}(1)\right)+\sum_{\substack{0 \leq k \leq m-2: \\ w_{k+1}=0}} \frac{1}{2^{k}} S_{\eta}^{k}(1)\right) .
$$

If $m=2$, then by (26) we find $\frac{1}{C}=\frac{2}{3 \eta}$ and $w_{1}=1$, so for all $\eta \in J_{11}$,

$$
\mu_{\eta}\left(\left[-\frac{1}{2}, \frac{1}{2}\right]\right)=\frac{1}{C}(1+\eta-1)=\frac{2}{3} .
$$

Assume $m \geq 3$. Use the notation $w_{i}^{j}=w_{i} \cdots w_{j}$ and recall from (10) that for $k \leq m$ we have $S_{\eta}^{k}(1)=2^{k}-2^{k} v\left(w_{1}^{k}\right) \eta$. Then

$$
\mu_{\eta}\left(\left[-\frac{1}{2}, \frac{1}{2}\right]\right)=\frac{1}{C}\left(\eta+\sum_{\substack{1 \leq k \leq m-2: \\ w_{k+1=1}}}\left(\frac{\eta}{2^{k}}-1+v\left(w_{1}^{k}\right) \eta\right)+\sum_{\substack{1 \leq k \leq m-2: \\ w_{k+1=0}}}\left(1-v\left(w_{1}^{k}\right) \eta\right)\right)
$$

Let

$$
\eta(w)=\#\left\{2 \leq k \leq m-1: w_{k}=0\right\}-\#\left\{2 \leq k \leq m-1: w_{k}=1\right\} .
$$

Then

$$
\begin{aligned}
\mu_{\eta}\left(\left[-\frac{1}{2}, \frac{1}{2}\right]\right) \\
\quad=\frac{1}{C}\left(\eta+\eta(w)+\eta\left(\sum_{\substack{1 \leq k \leq m-2: \\
w_{k+1=1}}}\left(\frac{1}{2^{k}}+v\left(w_{1}^{k}\right)\right)-\sum_{\substack{1 \leq k \leq m-2: \\
w_{k+1=0}}} v\left(w_{1}^{k}\right)\right)\right) \\
\quad=\frac{2^{m-1}}{2^{m}-1}\left(\frac{\eta(w)}{\eta}+2 v\left(w_{1}^{m-1}\right)+\sum_{\substack{1 \leq k \leq m-2: \\
w_{k+1=1}}} v\left(w_{1}^{k}\right)-\sum_{\substack{1 \leq k \leq m-2: \\
w_{k+1=0}}} v\left(w_{1}^{k}\right)\right),
\end{aligned}
$$


where we have used that $\sum_{1 \leq k \leq m-2:} \frac{1}{2^{k}}=\sum_{1 \leq k \leq m-2} \frac{w_{k+1}}{2^{k}}=2 v\left(w_{1}^{m-1}\right)-1$. If $w_{k+1}=1$, then $v\left(w_{1}^{k+1}\right)=v\left(w_{1}^{k}\right)+\frac{1}{2^{k+1}}$ and if $w_{k+1}=0$, then $v\left(w_{1}^{k+1}\right)=v\left(w_{1}^{k}\right)$.

This gives

$\mu_{\eta}\left(\left[-\frac{1}{2}, \frac{1}{2}\right]\right)=\frac{2^{m-1}}{2^{m}-1}\left(\frac{\eta(w)}{\eta}+v\left(w_{1}^{m-1}\right)+\sum_{\substack{1 \leq k \leq m-1: \\ w_{k}=1}} v\left(w_{1}^{k}\right)-\sum_{\substack{2 \leq k \leq m-1: \\ w_{k}=0}} v\left(w_{1}^{k}\right)\right)$.

For any primitive word $w_{1} \cdots w_{m}(m \geq 3)$ the expression

$$
K_{w}:=v\left(w_{1}^{m-1}\right)+\sum_{\substack{1 \leq k \leq m-1: \\ w_{k}=1}} v\left(w_{1}^{k}\right)-\sum_{\substack{1 \leq k \leq m-1: \\ w_{k}=0}} v\left(w_{1}^{k}\right)
$$

has a constant value on $J_{w}$. As a result

$$
\mu_{\eta}\left(\left[-\frac{1}{2}, \frac{1}{2}\right]\right)=\frac{2^{m-1}}{2^{m}-1}\left(\frac{\eta(w)}{\eta}+K_{w}\right),
$$

as a function of $\eta$ on the interval $J_{w}$, is increasing for $\eta(w)<0$, decreasing for $\eta(w)>0$ and constant if $\eta(w)=0$. Note that using (30) we can for any $\eta \in$ $\left(1, \frac{3}{2}\right) \backslash \mathcal{N}$ explicitly calculate the frequency of the digit 0 in the signed binary expansion $\left(d_{\eta, n}(x)\right)_{n \geq 1}$ for typical $x$. We now have Theorem B.

Proof of Theorem B. The continuity of $\eta \mapsto \mu_{\eta}\left(\left[-\frac{1}{2}, \frac{1}{2}\right]\right)$ is proved in Corollary 4.1, and the other two statements of the theorem follow from (30).

\section{$\S 5$. The maximal frequency of the digit 0}

It remains to show that the map $\eta \mapsto \mu_{\eta}\left(\left[-\frac{1}{2}, \frac{1}{2}\right]\right)$ takes its maximal value $\frac{2}{3}$ exactly on $\left[\frac{6}{5}, \frac{3}{2}\right]$. Figure 4 shows $\mu_{\eta}\left(\left[-\frac{1}{2}, \frac{1}{2}\right]\right)$ for several primitive words $w$. The value on the big plateau in the middle is obtained easily from the relation with the $\alpha$-continued fraction maps as follows.

From (30) it follows that the monotonicity behaviour of $\eta \mapsto \mu_{\eta}\left(\left[-\frac{1}{2}, \frac{1}{2}\right]\right)$ equals that of the entropy function $\alpha \mapsto h_{\nu_{\alpha}}\left(T_{\alpha}\right)$ for $\alpha$-continued fractions as described in (17). To see this, let $w=1^{a_{1}} \cdots 1^{a_{2 n+1}}$ be a primitive word. Then by Proposition 3.3 the quadratic interval $I_{a}$ is maximal with $a=\left[0 ; a_{1} \cdots a_{2 n+1}\right]$. For $N=1+\sum_{j \text { even }} a_{j}$ and $M=-1+\sum_{j \text { odd }} a_{j}$ we have $N-M=\eta(w)$. Therefore, by (17) we get that $\mu_{\eta}\left(\left[-\frac{1}{2}, \frac{1}{2}\right]\right)$ increases, decreases or is constant on $J_{w}$ if and only if $h_{\nu_{\alpha}}\left(T_{\alpha}\right)$ increases, decreases or is constant on $I_{a(w)}$ respectively. As mentioned in Section 3.1 it was shown in [KSS12, Sect. 10] that the entropy function $\alpha \mapsto$ $h_{\nu_{\alpha}}\left(T_{\alpha}\right)$ is constant on the interval $\left[g^{2}, g\right]$, where $g=\frac{\sqrt{5}-1}{2}$. Since $\frac{1}{\varphi(g)}=\frac{3}{2}$ and $\frac{1}{\varphi\left(g^{2}\right)}=\frac{6}{5}$, we have $\mu_{\eta}\left(\left[-\frac{1}{2}, \frac{1}{2}\right]\right)=\mu_{\frac{3}{2}}\left(\left[-\frac{1}{2}, \frac{1}{2}\right]\right)=\frac{2}{3}$ for any $\eta \in\left[\frac{6}{5}, \frac{3}{2}\right]$. This gives the plateau on the top of the graph in Figure 4. 
To prove Theorem $\mathrm{C}$ we still need to show that $\mu_{\eta}\left(\left[-\frac{1}{2}, \frac{1}{2}\right]\right)<\frac{2}{3}$ for all other values of $\eta$. We know from (12) that this holds for $\eta>\frac{3}{2}$. The proof of Theorem $\mathrm{C}$ requires the following lemma on the value of $\mu_{\eta}\left(\left[-\frac{1}{2}, \frac{1}{2}\right]\right)$ on the cascades of matching intervals.

Lemma 5.1. Let $w$ be a primitive word. Then $\eta\left(\psi^{n}(w)\right)=0$ for all $n \geq 1$ and the frequency function $\eta \mapsto \mu_{\eta}\left(\left[-\frac{1}{2}, \frac{1}{2}\right]\right)$ is constant on the interval $\left[p_{w}, L(w)\right]$.

Proof. Let $w=w_{1} \cdots w_{m}$ be a primitive word and let $k$ denote the number of 0 s that occur in $w$. Then $\eta(w)=k-(m-2-k)=2 k+2-m$. Since $w_{m}=1$ and $1-w_{1}=0$, the number of 0 s in $\psi(w)$ is $k+1+(m-2-k)=m-1$. Hence, $\eta(\psi(w))=0$ and by the same reasoning $\eta\left(\psi^{n}(w)\right)=0$ for all $n$. By (30), $\eta \mapsto \mu_{\eta}\left(\left[-\frac{1}{2}, \frac{1}{2}\right]\right)$ is constant on each interval $J_{\psi^{n}(w)}$, so that by continuity

$$
\mu_{\eta}\left(\left[-\frac{1}{2}, \frac{1}{2}\right]\right)=\mu_{L(w)}\left(\left[-\frac{1}{2}, \frac{1}{2}\right]\right)
$$

for all $\eta \in\left[p_{w}, L(w)\right]$

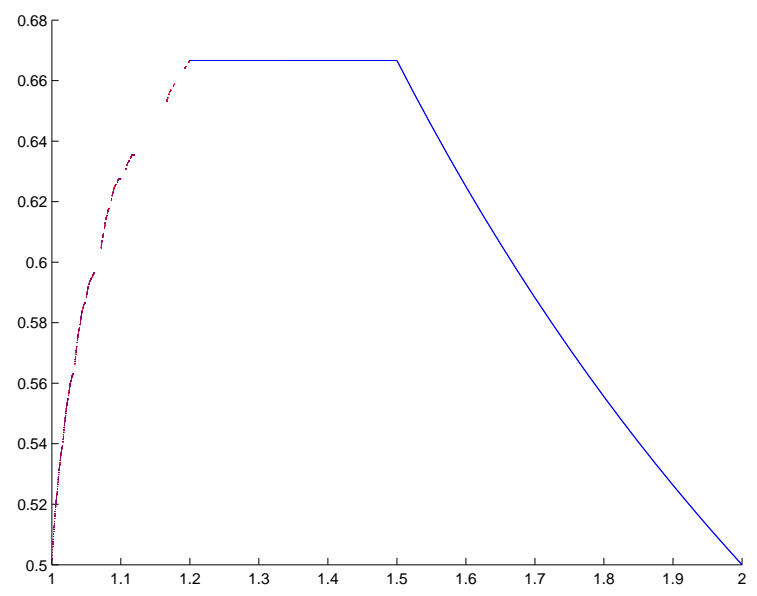

Figure 4 . The value of $\mu_{\eta}\left(\left[-\frac{1}{2}, \frac{1}{2}\right]\right)$ on all matching intervals $J_{w}$ with $m \leq 13$. The picture is made by Niels Langeveld.

Before we start on the proof of Theorem C, we consider the set of primitive words of the form $1^{m}$ separately in the next example. 
Example 5.1. Let $w=1^{m}$ with $m \geq 3$. Then $\eta(w)=-(m-2)$, so that the maximal value of $\mu_{\eta}\left(\left[-\frac{1}{2}, \frac{1}{2}\right]\right)$ is attained when $\frac{1}{\eta}$ is smallest, i.e., if

$$
\frac{1}{\eta}=v\left(\left(1^{m-1} 0\right)^{\infty}\right)=\frac{1}{2} \frac{1}{1-\frac{1}{2^{m}}}+\cdots+\frac{1}{2^{m-1}} \frac{1}{1-\frac{1}{2^{m}}}=\frac{1}{2^{m}-1} \sum_{k=1}^{m-1} 2^{k}=\frac{2^{m}-2}{2^{m}-1}
$$

For $1 \leq n \leq m-1$ we have $v\left(w_{1}^{n}\right)=\sum_{k=1}^{n} \frac{1}{2^{k}}=1-\frac{1}{2^{n}}$. Thus, for $\eta \in J_{w}$ it holds by (30) that

$$
\begin{aligned}
\mu_{\eta}\left(\left[-\frac{1}{2}, \frac{1}{2}\right]\right) & \leq \frac{2^{m-1}}{2^{m}-1}\left(-\frac{(m-2)\left(2^{m}-2\right)}{2^{m}-1}+1-\frac{1}{2^{m-1}}+\sum_{n=1}^{m-1}\left(1-\frac{1}{2^{n}}\right)\right) \\
& =\frac{2^{m-1}}{2^{m}-1}\left(-m+2+\frac{m-2}{2^{m}-1}+1-\frac{1}{2^{m-1}}+m-1-2+\frac{1}{2^{m-1}}\right) \\
& =\frac{2^{m-1}}{2^{m}-1} \frac{m-2}{2^{m}-1} .
\end{aligned}
$$

Note that this is less than $\frac{2}{3}$ if and only if $3(m-2) 2^{m-1}<2\left(2^{m}-1\right)^{2}$, which is if and only if

$$
m\left(2^{m}+2^{m-1}\right)+2^{m+2}<2^{2 m+1}+2^{m+1}+2^{m}+2 .
$$

This obviously holds for $m=3$. For $m>3$ we have $2 m-1>m+2$ and of course $m<2^{m}$, so

$m\left(2^{m}+2^{m-1}\right)+2^{m+2}<2^{2 m}+2^{2 m-1}+2^{m+2}<2^{2 m+1}<2^{2 m+1}+2^{m+1}+2^{m}+2$.

Hence, also for $m>3$ and any $\eta \in J_{1^{m}}$ we have $\mu_{\eta}\left(\left[-\frac{1}{2}, \frac{1}{2}\right]\right)<\frac{2}{3}$. Then the same statement holds on the cascade of matching intervals attached to $J_{1^{m}}$, i.e., for any $\eta \in J_{\psi^{n}\left(1^{m}\right)}, n \geq 1$.

Proof of Theorem C. To prove Theorem C it is enough to consider $\eta \in\left(1, \frac{6}{5}\right)$. Corollary 4.1 then implies that we need to consider only values of $\eta$ in a matching interval $J_{w}$ for which $w$ is primitive and $w 0^{\infty} \succ 1(10)^{\infty}$. The formula obtained in (30) shows that whether on $J_{w}$ the frequency map $\eta \mapsto \mu_{\eta}\left(\left[-\frac{1}{2}, \frac{1}{2}\right]\right)$ is increasing, decreasing or constant depends on the value of $\eta(w)$. By Proposition 3.4 and Lemma 5.1 it follows that attached to each matching interval there is a cascade of matching intervals on which the frequency map is constant. Hence, by Corollary 4.1 , to prove that $\mu_{\eta}\left(\left[-\frac{1}{2}, \frac{1}{2}\right]\right)<\frac{2}{3}$ for any $\eta \in\left(1, \frac{6}{5}\right)$ it is enough to prove that this is the case for any $\eta$ in a matching interval $J_{w}$ that satisfies $\eta(w)=0$. This is what we will do.

Let $w=w_{1} \cdots w_{m}=1^{a_{1}} 0^{a_{2}} \cdots 1^{a_{2 n+1}}$ be a primitive word with $\eta(w)=0$. Then $m$ is even and

$$
a_{1}+\cdots+a_{2 n+1}-2=\frac{m-2}{2}=a_{2}+\cdots+a_{2 n} .
$$


Moreover, by Example 5.1 we can assume that $w=1^{a_{1}} 0 w_{a_{1}+2} \cdots w_{m}$, so that $m \geq a_{1}+2$. To give precise estimates on $\mu_{\eta}\left(\left[-\frac{1}{2}, \frac{1}{2}\right]\right)$ we rewrite (29) to obtain

$$
\begin{aligned}
\mu_{\eta}\left(\left[-\frac{1}{2}, \frac{1}{2}\right]\right)=\frac{2^{m-1}}{2^{m}-1}\left(2 v\left(w_{1}^{m-1}\right)\right. & +\sum_{\substack{1 \leq \ell \leq m-2: \\
w_{\ell+1=1}}}\left(v\left(w_{1}^{\ell}\right)-\frac{1}{\eta}\right) \\
& \left.+\sum_{\substack{1 \leq \ell \leq m-2: \\
w_{\ell+1=0}}}\left(\frac{1}{\eta}-v\left(w_{1}^{\ell}\right)\right)\right) .
\end{aligned}
$$

Set $s_{0}=0$ and for $1 \leq k \leq 2 n+1, s_{k}=a_{1}+\cdots+a_{k}$. Since $\mu_{\eta}\left(\left[-\frac{1}{2}, \frac{1}{2}\right]\right)$ is the same for all $\eta \in J_{w}$, we take $\eta=\frac{1}{v(w)}$. Note that for any $k$ the $k$ th block of $1 \mathrm{~s}$ in $w$ satisfies

$$
\sum_{j=s_{2 k}+1}^{s_{2 k+1}} \frac{1}{2^{j}}=\frac{1}{2^{s_{2 k}}}-\frac{1}{2^{s_{2 k+1}}}=\frac{2^{a_{2 k+1}}-1}{2^{s_{2 k+1}}},
$$

so that

$$
\sum_{\substack{1 \leq \ell \leq m-2: \\ w_{\ell+1=0}}}\left(v(w)-v\left(w_{1}^{\ell}\right)\right)=\sum_{j=1}^{n} \frac{2^{a_{2 j+1}}-1}{2^{s_{2 j+1}}}\left(a_{2}+\cdots+a_{2 j}\right) .
$$

On the other hand,

$$
\begin{aligned}
\sum_{\substack{1 \leq \ell \leq m-2: \\
w_{\ell+1}}}\left(v(w)-v\left(w_{1}^{\ell}\right)\right) & =\sum_{2 \leq \ell+1 \leq s_{1}} \frac{1}{2^{\ell}} v\left(w_{\ell+1}^{m}\right)+\sum_{s_{2}+1 \leq \ell+1 \leq s_{3}} \frac{1}{2^{\ell}} v\left(w_{\ell+1}^{m}\right)+\cdots+\sum_{s_{2 n}+1 \leq \ell \leq s_{2 n+1}-1} \frac{1}{2^{\ell}} v\left(w_{\ell+1}^{m}\right) \\
= & 2\left(v(w)-\frac{1}{2}-\frac{1}{2^{m}}\right)-\frac{a_{1}-1}{2^{s_{1}}}-\frac{a_{3}}{2^{s_{3}}}-\cdots-\frac{a_{2 n-1}}{2^{s_{2 n-1}}}-\frac{a_{2 n+1}-1}{2^{s_{2 n+1}}} \\
& +\sum_{j=1}^{n}\left(a_{1}+a_{3}+\cdots+a_{2 j-1}-1\right) \frac{2^{a_{2 j+1}}-1}{2^{s_{2 j+1}}} \\
= & 2 v(w)-1-\frac{1}{2^{m-1}} \\
& +\sum_{j=1}^{n} \frac{\left(a_{1}+a_{3}+\cdots+a_{2 j-1}-1\right)\left(2^{a_{2 j+1}}-1\right)-a_{2 j+1}}{2^{s_{2 j+1}}}-\frac{a_{1}-1}{2^{a_{1}}} .
\end{aligned}
$$

Inserting this into (31) and using that $2 v\left(w_{1}^{m-1}\right)=2 v(w)-\frac{1}{2^{m-1}}$ we get that the inequality

$\mu_{\eta}\left(\left[-\frac{1}{2}, \frac{1}{2}\right]\right)=\frac{2^{m-1}}{2^{m}-1}\left(1+\frac{a_{1}-1}{2^{a_{1}}}+\sum_{j=1}^{n} \frac{a_{2 j+1}+\left(2^{a_{2 j+1}}-1\right)\left(1+\sum_{\ell=1}^{2 j}(-1)^{\ell} a_{\ell}\right)}{2^{s_{2 j+1}}}\right)<\frac{2}{3}$ 
holds if and only if

$$
2^{m+1}+2^{m}+2^{2}+3\left(a_{1}-1\right) 2^{m-a_{1}}+3 \sum_{j=1}^{n} R_{j}(w)<2^{m+2},
$$

where we have put

$R_{j}(w)=a_{2 j+1} 2^{m-s_{2 j+1}}+2^{m-s_{2 j}}\left(1+\sum_{\ell=1}^{2 j}(-1)^{\ell} a_{\ell}\right)+2^{m-s_{2 j+1}}\left(\sum_{\ell=1}^{2 j}(-1)^{\ell+1} a_{\ell}-1\right)$.

We split the proof into several cases according to the value of $a_{1}$.

(I) $a_{1} \geq 3$ : Consider first the term $3\left(a_{1}-1\right) 2^{m-a_{1}}$. For $a_{1}=4$ and $a_{1}=3$ we can just calculate this and for $a_{1} \geq 5$ we can use the fact that $p \leq 2^{\frac{p}{2}}$ for any $p \geq 4$ to obtain

$$
3\left(a_{1}-1\right) 2^{m-a_{1}} \leq \begin{cases}2^{m-2}+2^{m-3} & \text { if } a_{1} \geq 5 \\ 2^{m-1}+2^{m-4} & \text { if } a_{1}=4 \\ 2^{m-1}+2^{m-2} & \text { if } a_{1}=3 .\end{cases}
$$

Next consider $R_{1}(w)$. By primitivity we have $a_{1}>a_{2}$. For $a_{1}=3$ we can make precise estimates by considering the cases $a_{2}=1$ and $a_{2}=2$ separately. For $a_{2}=1$ we have $a_{1}-a_{2}-1=1$ and using the fact that $p \leq 2^{p-1}$ for all $p \geq 1$ gives

$$
3 R_{1}(w)=3\left(a_{3} 2^{m-s_{3}}-2^{m-s_{2}}+2^{m-s_{3}}\right) \leq 3\left(2^{m-s_{3}+a_{3}}-2^{m-s_{2}}\right)=0 .
$$

In the case $a_{2}=2$ we get $a_{1}-a_{2}-1=0$ and thus

$$
3 R_{1}(w)=3 a_{3} 2^{m-a_{3}} \leq 3 \cdot 2^{m-s_{2}-1}=2^{m-5}+2^{m-6} .
$$

For $a_{1} \geq 4$, we get, again by using that $p \leq 2^{p-1}$ for all $p \geq 1$,

$$
3 R_{1}(w) \leq 3 \cdot 2^{m-s_{3}}\left(a_{1}-a_{2}+a_{3}-1\right) \leq 3 \cdot 2^{m-2 a_{2}-2} \leq 2^{m-3}+2^{m-4} .
$$

So, we have obtained

$$
3 R_{1}(w) \leq \begin{cases}2^{m-3}+2^{m-4} & \text { if } a_{1} \geq 4 \\ 2^{m-5}+2^{m-6} & \text { if } a_{1}=3\end{cases}
$$

Now consider $R_{j}(w)$ for $2 \leq j \leq n$. Note that the terms $1+\sum_{\ell=1}^{2 j}(-1)^{\ell} a_{\ell}$ and $\sum_{\ell=1}^{2 j}(-1)^{\ell+1} a_{\ell}-1$ have opposite signs, so that to get an upper bound it is enough to consider only the positive term. First assume that $1+\sum_{\ell=1}^{2 j}(-1)^{\ell} a_{\ell}>0$. Then, 
since $p \leq 2^{p-1}$ for all $p \geq 1$ and $s_{k} \geq a_{1}+k-1$ for all $k$, we get

$$
\begin{aligned}
R_{j}(w) & \leq a_{2 j+1} 2^{m-s_{2 j+1}}+2^{m-s_{2 j}}\left(1+\sum_{\ell=1}^{2 j}(-1)^{\ell} a_{\ell}\right) \\
& \leq 2^{m-s_{2 j}-1}+2^{m-2\left(a_{1}+a_{3}+\cdots+a_{2 j-1}\right)} \leq 2^{m-a_{1}-2 j}+2^{m-2 a_{1}-2 j+2} .
\end{aligned}
$$

On the other hand, if $-1+\sum_{\ell=1}^{2 j}(-1)^{\ell+1} a_{\ell}>0$, then

$$
\begin{aligned}
R_{j}(w) & \leq a_{2 j+1} 2^{m-s_{2 j+1}}+2^{m-s_{2 j+1}}\left(-1+\sum_{\ell=1}^{2 j}(-1)^{\ell+1} a_{\ell}\right) \\
& \leq 2^{m-s_{2 j}-1}+2^{m-2\left(a_{2}+a_{4}+\cdots+a_{2 j}+1\right)} \leq 2^{m-a_{1}-2 j}+2^{m-2 j-2} .
\end{aligned}
$$

So, in all cases we have $R_{j}(w) \leq 2^{m-a_{1}-2 j}+2^{m-2 j-2}$. Then, since $a_{1} \geq 3$,

$$
\begin{aligned}
3 \sum_{j=2}^{n} R_{j}(w) & \leq 3 \sum_{j=2}^{n}\left(2^{m-3-2 j}+2^{m-2-2 j}\right) \\
& =3 \sum_{j=6}^{3+2 n} 2^{m-j}<3 \cdot 2^{m-5}=2^{m-4}+2^{m-5}
\end{aligned}
$$

Putting (33), (34) and (35) together leads to the following. For $a_{1} \geq 5$ it follows from $\eta(w)=0$ that $m \geq 10$, so that

$$
\begin{aligned}
2^{m+1} & +2^{m}+2^{2}+3\left(a_{1}-1\right) 2^{m-a_{1}}+3 \sum_{j=1}^{n} R_{j}(w) \\
& \leq 2^{m+1}+2^{m}+2^{2}+2^{m-2}+2^{m-3}+2^{m-3}+2^{m-4}+2^{m-4}+2^{m-5} \\
& =2^{m+1}+2^{m}+2^{m-1}+2^{m-3}+2^{m-5}+2^{2}<2^{m+2} .
\end{aligned}
$$

For $a_{1}=4$ we have $m \geq 8$ and

$$
\begin{aligned}
2^{m+1} & +2^{m}+2^{2}+3\left(a_{1}-1\right) 2^{m-a_{1}}+3 \sum_{j=1}^{n} R_{j}(w) \\
& \leq 2^{m+1}+2^{m}+2^{2}+2^{m-1}+2^{m-4}+2^{m-3}+2^{m-4}+2^{m-4}+2^{m-5} \\
& =2^{m+1}+2^{m}+2^{m-1}+2^{m-2}+2^{m-4}+2^{m-5}+2^{2}<2^{m+2} .
\end{aligned}
$$

For $a_{1}=3$ we have $m \geq 6$ and get

$$
\begin{aligned}
& 2^{m+1}+2^{m}+2^{2}+3\left(a_{1}-1\right) 2^{m-a_{1}}+3 \sum_{j=1}^{n} R_{j}(w) \\
& \leq 2^{m+1}+2^{m}+2^{2}+2^{m-1}+2^{m-2}+2^{m-5}+2^{m-6}+2^{m-4}+2^{m-5} \\
& =2^{m+1}+2^{m}+2^{m-1}+2^{m-2}+2^{m-3}+2^{m-6}+2^{2}<2^{m+2} .
\end{aligned}
$$


Hence, in all cases the inequality from (32) is satisfied and $\mu_{\eta}\left(\left[-\frac{1}{2}, \frac{1}{2}\right]\right)<\frac{2}{3}$.

(II) $a_{1}=2$ : In this case $w$ starts with 1101 and cannot have more than two consecutive 0 s or 1 s. Since $\eta<\frac{6}{5}$ we have $w 0^{\infty} \succ 1(10)^{\infty}$, so there is some $k \geq 1$, such that

$$
w=1(10)^{k} 110 w_{2 k+5} \cdots w_{m}
$$

Since $a_{2 j+1} 2^{m-s_{2 j}+1}=2^{m-s_{2 j}-1}$ for all $1 \leq j \leq n$ and $a_{1}=a_{2}+1$ we have

$$
R_{j}(w)=2^{m-s_{2 j}-1}+2^{m-s_{2 j}}\left(1-2^{-a_{2 j+1}}\right)\left(-a_{3}+a_{4}-\cdots-a_{2 j-1}+a_{2 j}\right) .
$$

We further know that $3\left(a_{1}-1\right) 2^{m-a_{1}}=2^{m-1}+2^{m-2}$. Since for any $2 \leq j \leq k$ we have $-a_{3}+a_{4}-\cdots-a_{2 j-1}+a_{2 j}=0$, it follows that

$$
3 \sum_{j=2}^{k} R_{j}(w)=3 \sum_{j=2}^{k} 2^{m-s_{2 j}-1}=\sum_{j=5}^{2 k+2} 2^{m-j} .
$$

Hence, to show that inequality (32) holds we would like to have an upper bound for

$$
\begin{aligned}
Q(w)= & \sum_{j=-1}^{2 k+2} 2^{m-j}+2^{2} \\
& +3 \sum_{j=k+1}^{n}\left(2^{m-s_{2 j}-1}+2^{m-s_{2 j}}\left(1-2^{-a_{2 j+1}}\right)\left(-a_{3}+a_{4}-\cdots-a_{2 j-1}+a_{2 j}\right)\right) .
\end{aligned}
$$

The terms $-a_{3}+a_{4}-\cdots-a_{2 j-1}+a_{2 j}$ are largest if we see blocks 00 before we see blocks 11. Since $\psi\left(1(10)^{k} 11\right)=1(10)^{k} 110(01)^{k+1}$, primitivity implies that each occurrence of 00 must be followed by $(10)^{k}$ or $(10)^{j} 11$ for some $j<k$. This in turn implies that there is no primitive word $w$ with $\eta(w)=0$ and $m<2(2 k+3)$. Hence, we consider only words of even length at least equal to $2(2 k+3)$ and for each such word $w$ there is a unique $p \geq 1$, such that its length $m$ satisfies $(p+1)(2 k+3) \leq m<(p+2)(2 k+3)$. We consider a number of cases.

If $p$ is odd, then

$$
Q(w) \leq \begin{cases}Q\left(1(10)^{k} 11\left(0(01)^{k+1}\right)^{p}\right) & \text { if } m=(p+1)(2 k+3) \\ Q\left(1(10)^{k} 11\left(0(01)^{k+1}\right)^{p} 0(01)^{i} 1\right) & \text { if } m=(p+1)(2 k+3)+2 i+2 \\ & 0 \leq i \leq k\end{cases}
$$

If $p$ is even instead, then

$$
Q(w) \leq \begin{cases}Q\left(1(10)^{k} 11\left(0(01)^{k+1}\right)^{p} 1\right) & \text { if } m=(p+1)(2 k+3)+1 \\ Q\left(1(10)^{k} 11\left(0(01)^{k+1}\right)^{p} 0(01)^{i}\right) & \text { if } m=(p+1)(2 k+3)+2 i+1 \\ & 0 \leq i \leq k\end{cases}
$$


To begin we compute an upper bound for $Q\left(w^{\diamond}\right)$, where $w^{\diamond}=1(10)^{k} 11\left(0(01)^{k+1}\right)^{p}$.

It holds that $a_{2 j+1}=1$ for all $j \geq k+1$, so that we can rewrite $Q\left(w^{\diamond}\right)$ as

(36) $Q\left(w^{\diamond}\right)=\sum_{j=-1}^{2 k+2} 2^{m-j}+2^{2}+3 \sum_{j=k+1}^{n} 2^{m-s_{2 j}-1}\left(1-a_{3}+a_{4}-\cdots-a_{2 j-1}+a_{2 j}\right)$.

For each $1 \leq j \leq p$ and $0 \leq \ell \leq k$ we have

$$
-a_{3}+a_{4}-\cdots-a_{2 j k+2 j+2 \ell-1}+a_{2 j k+2 j+2 \ell}=j-1
$$

and $s_{2 j k+2 j+2 \ell}=2 j k+3 j+2 \ell+3$. Putting this in (36) gives

$$
\begin{aligned}
Q\left(w^{\diamond}\right) & =\sum_{j=-1}^{2 k+2} 2^{m-j}+2^{2}+3 \sum_{j=1}^{p} j \sum_{\ell=0}^{k} 2^{m-2 j k-3 j-2 \ell-3} \\
& =\sum_{j=-1}^{2 k+2} 2^{m-j}+2^{2}+3 \sum_{\ell=0}^{k} 2^{m-2 \ell-3} \sum_{j=1}^{p} j 2^{-(2 k+3) j} .
\end{aligned}
$$

Since

$$
\sum_{j=1}^{p} j 2^{-(2 k+3) j}<\sum_{j=1}^{\infty}(j+1) 2^{-(2 k+3)}-\sum_{j=1}^{\infty} 2^{-(2 k+3) j}=\frac{2^{2 k+3}}{\left(2^{2 k+3}-1\right)^{2}}<\frac{1}{2^{2 k+2}},
$$

we have

$$
3 \sum_{\ell=0}^{k} 2^{m-2 \ell-3} \sum_{j=1}^{p} j 2^{-(2 k+3) j}<2^{m-2 k-5}\left(4-\frac{1}{2^{2 k}}\right)<2^{m-2 k-3} .
$$

From $p \geq 1$ it follows that $m=(p+1)(2 k+3) \geq 2(2 k+3)$, so that

$$
Q\left(w^{\diamond}\right)<\sum_{j=-1}^{2 k+3} 2^{m-j}+2^{2}<2^{m+2}
$$

Now consider $Q\left(w^{\boldsymbol{\bullet}}\right)$, where $w^{\boldsymbol{\bullet}}=1(10)^{k} 11\left(0(01)^{k+1}\right)^{p} 0(01)^{i} 1$ for some $0 \leq i \leq k$. Then $a_{2 j+1}=1$ for all $k+1 \leq j<n$ and $a_{2 n+1}=2$. With the same computations as above this gives

$$
\begin{aligned}
Q\left(w^{\mathbf{\omega}}\right)= & \sum_{j=-1}^{2 k+2} 2^{m-j}+2^{2}+3 \sum_{j=1}^{p} j \sum_{\ell=0}^{k} 2^{m-2 j k-3 j-2 \ell-3} \\
& +3 \sum_{\ell=0}^{i-1}(p+1) 2^{m-2(p+1) k-3(p+1)-2 \ell-3} \\
& +3 \cdot 2^{m-2(p+1) k-3(p+1)-2 i-3}+3 p \cdot 2^{m-2(p+1) k-3(p+1)-2 i-2}\left(1-2^{-2}\right)
\end{aligned}
$$




$$
\begin{aligned}
\leq & \sum_{j=-1}^{2 k+2} 2^{m-j}+2^{2} \\
& +3 \sum_{j=1}^{p+1} j \sum_{\ell=0}^{k} 2^{m-2 j k-3 j-2 \ell-3}+3 p \cdot 2^{m-2(p+1) k-3(p+1)-2 i-4} .
\end{aligned}
$$

Using (37) and the fact that $p \leq 2^{p-1}$ we get

$$
Q\left(w^{\mathbf{\uparrow}}\right)<\sum_{j=-1}^{2 k+3} 2^{m-j}+2^{2}+2^{m-2(p+1) k-2 p-2 i-7}+2^{m-2(p+1) k-2 p-2 i-8} .
$$

Since $p \geq 1$ we obtain $2(p+1) k+2 p+2 i+7 \geq 4 k+9>2 k+5$ and it follows that $Q\left(w^{\boldsymbol{\Phi}}\right)<2^{m+2}$. Next consider $Q\left(w^{\odot}\right)$, where $w^{\odot}=1(10)^{k} 11\left(0(01)^{k+1}\right)^{p} 1$, so that $m=2 p k+3 p+2 k+4$. Then again we have $a_{2 j+1}=1$ for all $k+1 \leq j<n$ and $a_{2 n+1}=2$. In the same way as above this gives

$$
\begin{aligned}
Q\left(w^{\circlearrowright}\right)= & \sum_{j=-1}^{2 k+2} 2^{m-j}+2^{2}+3 \sum_{j=1}^{p-1} j \sum_{\ell=0}^{k} 2^{m-2 j k-3 j-2 \ell-3}+3 \sum_{\ell=0}^{k-1} p 2^{m-2 p k-3 p-2 \ell-3} \\
& +3 \cdot 2^{m-2 p k-3 p-2 k-3}+3(p-1) \cdot 2^{m-2 p k-3 p-2 k-2}\left(1-2^{-2}\right) \\
< & \sum_{j=-1}^{2 k+3} 2^{m-j}+2^{2}+3(p-1) \cdot 2^{m-2 p k-3 p-2 k-3} .
\end{aligned}
$$

Since $p \geq 2$, we use $p-1 \leq 2^{p-2}$ to obtain

$$
Q\left(w^{\odot}\right)<\sum_{j=-1}^{2 k+3} 2^{m-j}+2^{2}+2^{m-2 p k-2 p-2 k-4}+2^{m-2 p k-2 p-2 k-5}<2^{m+2} .
$$

Finally, consider $Q\left(w^{\mathbf{4}}\right)$, where $w^{\boldsymbol{\mu}}=1(10)^{k} 11\left(0(01)^{k+1}\right)^{p} 0(01)^{i}$ for some $0 \leq i \leq$ $k$. Then $a_{2 j+1}=1$ for all $j \geq k+1$, so that

$$
\begin{aligned}
Q\left(w^{\mathbf{4}}\right)= & \sum_{j=-1}^{2 k+2} 2^{m-j}+2^{2}+3 \sum_{j=1}^{p} j \sum_{\ell=0}^{k} 2^{m-2 j k-3 j-2 \ell-3} \\
& +3 \sum_{\ell=0}^{i}(p+1) 2^{m-2(p+1) k-3(p+1)-2 \ell-3} \\
< & \sum_{j=-1}^{2 k+3} 2^{m-j}+2^{2}<2^{m+2}
\end{aligned}
$$

Since we have considered all possible values of $m$, this finishes the proof. 


\section{Appendix. Proof of Proposition 3.2}

The content of this section is solely due to D. Kong [Kon]; it gives the proof of Proposition 3.2. For ease of notation we denote $\overline{w_{1} \ldots w_{n}}=\left(1-w_{1}\right) \ldots\left(1-w_{n}\right)$.

Proof of Proposition 3.2. Suppose $w=w_{1} \cdots w_{m}$ is primitive. To show that $w$ is admissible, by Definition 3.1(ii) it suffices to show that

$$
\overline{w_{1} \ldots w_{m-k}} \prec w_{k+1} \ldots w_{m} \quad \text { for all } 0 \leq k \leq m-1 \text {. }
$$

Since $w_{m}=1$, (38) holds for $k=0$. Suppose (38) fails for some minimal $1 \leq k \leq$ $m-1$. Then $w_{k+1} \ldots w_{m} \preceq \overline{w_{1} \ldots w_{m-k}}$. By the minimality of $k$ we have $w_{k}=1$. Then by using $w_{m}=1$ it follows that

$$
\begin{aligned}
w_{1} \ldots w_{k} 0^{\infty} \prec w_{1} \ldots w_{m} 0^{\infty} & \preceq w_{1} \ldots w_{k} \overline{w_{1} \ldots w_{m-k}} 0^{\infty} \\
& \prec w_{1} \ldots w_{k} \overline{w_{1} \ldots w_{k-1}} 10^{\infty}
\end{aligned}
$$

which contradicts primitivity. Hence, $w_{1} \ldots w_{m}$ is an admissible word. Conversely, assume $w_{1} \cdots w_{m}$ is admissible. Then for any $0 \leq k \leq m-1$ we have

$$
\overline{w_{1} \ldots w_{m-k}} \prec w_{k+1} \ldots w_{m} \preceq w_{1} \ldots w_{m-k} .
$$

So to prove the result, we need only to check conditions (i) and (iii) of Definition 3.1. Taking $k=m-1$ in (39) it follows that $w_{1}=w_{m}=1$. If $m=2$, then $w_{1}=w_{2}=w_{m}=1$. Let $m \geq 3$ and assume $w_{2}=0$; then by (39) it follows that

$$
w_{1} \ldots w_{m}=(10)^{\ell} 1 \quad \text { with } \ell \geq 1 \text {, }
$$

leading to a contradiction with (39) since $w_{2} \ldots w_{m}=(01)^{\ell}=\overline{w_{1} \ldots w_{m-1}}$. Therefore, $w_{2}=1$. It remains to prove condition (iii) of Definition 3.1. Suppose it fails. Then there exists a word $b_{1} \ldots b_{j}$ such that

$$
b_{1} \ldots b_{j} 0^{\infty} \prec w_{1} \ldots w_{m} 0^{\infty} \prec b_{1} \ldots b_{j} \overline{b_{1} \ldots b_{j-1}} 10^{\infty}
$$

We can assume with no loss of generality that $b_{j}=1$. Then by (40) we have $j<m$ and $b_{1} \ldots b_{j}=w_{1} \ldots w_{j}$. Furthermore,

$$
\begin{array}{ll}
w_{j+1} \ldots w_{m} \preceq \overline{b_{1} \ldots b_{m-j}}=\overline{w_{1} \ldots w_{m-j}} & \text { if } m<2 j ; \\
w_{j+1} \ldots w_{2 j} \prec \overline{b_{1} \ldots b_{j-1}} 1=\overline{w_{1} \ldots w_{j-1}} 1 & \text { if } m \geq 2 j .
\end{array}
$$

If $m<2 j$, then $w_{j+1} \ldots w_{m} \preceq \overline{w_{1} \ldots w_{m-j}}$, leading to a contradiction with (39). If $m=2 j$, then

$$
w_{j+1} \ldots w_{m-j}=w_{j+1} \ldots w_{2 j} \preceq \overline{w_{1} \ldots w_{j}}=\overline{w_{1} \ldots w_{m-j}}
$$


again leading to a contradiction with (39). If $m>2 j$, then $w_{j+1} \ldots w_{2 j} \preceq \overline{w_{1} \ldots w_{j}}$. Setting $k=j$ in (39) gives

$$
w_{1} \ldots w_{2 j}=w_{1} \ldots w_{j} \overline{w_{1} \ldots w_{j}} .
$$

Again by iterations of (39) we conclude that

$$
w_{1} \ldots w_{m}=\left(w_{1} \ldots w_{j} \overline{w_{1} \ldots w_{j}}\right)^{\ell} w_{1} \ldots w_{p} \quad \text { with } \ell \geq 1 \text { and } 1 \leq p<2 j .
$$

Then

$$
w_{m-p-j+1} \ldots w_{m}=\overline{w_{1} \ldots w_{j}} w_{1} \ldots w_{p}=\overline{w_{1} \ldots w_{p+j}},
$$

leading to a contradiction with (39). Thus condition (iii) of Definition 3.1, and $w_{1} \ldots w_{m}$ is primitive.

\section{Acknowledgements}

The second author was partially supported by the NWO Veni-grant 639.031.140. We thank Niels Langeveld for producing Figure 4.

\section{References}

[AC83] J.-P. Allouche and M. Cosnard, Itérations de fonctions unimodales et suites engendrées par automates, C. R. Acad. Sci. Paris Sér. I Math. 296 (1983), 159-162. Zbl 0547.58027 MR 0693191

[AC01] J.-P. Allouche and M. Cosnard, Non-integer bases, iteration of continuous real maps, and an arithmetic self-similar set, Acta Math. Hungar. 91 (2001), 325-332. Zbl 1012.11007 MR 1912007

[BCIT13] C. Bonanno, C. Carminati, S. Isola and G. Tiozzo, Dynamics of continued fractions and kneading sequences of unimodal maps, Discrete Contin. Dyn. Syst. 33 (2013), 1313-1332. Zbl 1290.37018 MR 2995847

[BCK17] H. Bruin, C. Carminati and C. Kalle, Matching for generalised $\beta$-transformations, Indag. Math. (N.S.) 28 (2017), 55-73. Zbl 1360.37096 MR 3597033

[BCMP19] H. Bruin, C. Carminati, S. Marmi and A. Profeti, Matching in a family of piecewise affine maps, Nonlinearity 32 (2019), 172--208. Zbl 1408.37066 MR 3893724

[BSORG13] V. Botella-Soler, J. A. Oteo, J. Ros and P. Glendinning, Lyapunov exponent and topological entropy plateaus in piecewise linear maps, J. Phys. A 46 (2013), 125101, 26pp. Zbl 1267.37013 MR 3035348

[BG97] A. Boyarsky and P. Góra, Laws of chaos: Invariant measures and dynamical systems in one dimension, Probability and Its Applications, Birkhäuser, Boston, MA, 1997. Zbl 0893.28013 MR 1461536

[CMPT10] C. Carminati, S. Marmi, A. Profeti and G. Tiozzo, The entropy of $\alpha$-continued fractions: numerical results, Nonlinearity 23 (2010), 2429-2456. Zbl 1233.11083 MR 2672682

[CT12] C. Carminati and G. Tiozzo, A canonical thickening of $\mathbb{Q}$ and the entropy of $\alpha$ continued fraction transformations, Ergodic Theory Dynam. Systems 32 (2012), 1249-1269. Zbl 1268.37040 MR 2955313 
[CT13] C. Carminati and G. Tiozzo., Tuning and plateaux for the entropy of $\alpha$-continued fractions, Nonlinearity 26 (2013), 1049-1070. Zbl 1334.11062 MR 3040595

[CMO98] H. Cohen, A. Miyaji and T. Ono, Efficient elliptic curve exponentiation using mixed coordinates, in Advances in cryptology-ASIACRYPT' 98 (Beijing), Lecture Notes in Computer Science 1514, Springer, Berlin, 1998, 51-65. Zbl 0939.11039 MR 1726152

[CM18] D. Cosper and M. Misiurewicz, Entropy locking, Fund. Math. 241 (2018), 83-96. Zbl 1394.37069 MR 3756101

[DKL06] K. Dajani, C. Kraaikamp and P. Liardet, Ergodic properties of signed binary expansions, Discrete Contin. Dyn. Syst. 15 (2006), 87-119. Zbl 1115.37007 MR 2191387

[DKS09] K. Dajani, C. Kraaikamp and W. Steiner, Metrical theory for $\alpha$-Rosen fractions, J. Eur. Math. Soc. (JEMS), 11 (2009), 1259-1283. Zbl 1184.28016 MR 2557135

[Dek77] M. Dekking, Transcendance du nombre de Thue-Morse, C. R. Acad. Sci. Paris Sér. A-B 285 (1977), A157-A160. Zbl 0362.10028 MR 0457363

[EJK90] P. Erdös, I. Joó and V. Komornik, Characterization of the unique expansions $1=$ $\sum_{i=1}^{\infty} q^{-n_{i}}$ and related problems, Bull. Soc. Math. France 118 (1990), 377-390. Zbl 0721.11005 MR 1078082

[KL07] V. Komornik and P. Loreti, On the topological structure of univoque sets, J. Number Theory 122 (2007), 157-183. Zbl 1111.11005 MR 2287118

[Kon] D. Kong, Equivalence of primitivity and admissibility, Private communication.

[Kop90] C. Kopf, Invariant measures for piecewise linear transformations of the interval, Appl. Math. Comput. 39 (1990), 123-144. Zbl 0712.28007 MR 1071209

[KT93] K. Koyama and Y. Tsuruoka, Speeding up elliptic cryptosystems by using a signed binary window method, in Advances in cryptology - CRYPTO '92 (Santa Barbara, $C A, 1992)$, Lecture Notes in Computer Science 740, Springer, Berlin, 1993, 345-357. Zbl 0816.94016 MR 1287864

[KSS12] C. Kraaikamp, T. Schmidt and W. Steiner, Natural extensions and entropy of $\alpha$-continued fractions, Nonlinearity 25 (2012), 2207-2243. Zbl 1333.11079 MR 2946184

[MO90] F. Morain and J. Olivos, Speeding up the computations on an elliptic curve using addition-subtraction chains. RAIRO Inform. Théor. Appl. 24 (1990), 531-543. Zbl 0724.11068 MR 1082914

[Nak81] H. Nakada, Metrical theory for a class of continued fraction transformations and their natural extensions, Tokyo J. Math. 4 (1981), 399-426. Zbl 0479.10029 MR 0646050

[Nak] H. Nakada, On the maximum value of entropy of $\alpha$-continued fraction maps, talk at Numeration 2019, https://seafile.aau.at/d/ec9098f5e6a844abb957/files/?p= /Numeration2019-Nakada.pdf.

[NN08] H. Nakada and R. Natsui, The non-monotonicity of the entropy of $\alpha$-continued fraction transformations, Nonlinearity 21 (2008), 1207-1225. Zbl 1143.11024 MR 2422375

[Par60] W. Parry, On the $\beta$-expansions of real numbers, Acta Math. Acad. Sci. Hungar.11 (1960), 401-416. Zbl 0099.28103 MR 0142719 